\title{
Accurate Relative Location Estimates for the North Korean Nuclear Tests Using Empirical Slowness Corrections
}

\author{
S. J. Gibbons ${ }^{1}$, F. Pabian ${ }^{2}$, S. P. Näsholm ${ }^{1}$, T. Kværna ${ }^{1}$ and S. Mykkeltveit ${ }^{1}$ \\ ${ }^{1}$ NORSAR, P.O. Box 53, 2027 Kjeller, Norway, \\ E-mail: steven@norsar.no \\ ${ }^{2}$ Los Alamos National Laboratory.
}

\section{SUMMARY}

Declared North Korean nuclear tests in 2006, 2009, 2013, and 2016 were observed seismically at regional and teleseismic distances. Waveform similarity allows the events to be located relatively with far greater accuracy than the absolute locations can be determined from seismic data alone. There is now significant redundancy in the data given the large number of regional and teleseismic stations that have recorded multiple events, and relative location estimates can be confirmed independently by performing calculations on many mutually exclusive sets of measurements. Using a 1-dimensional global velocity model, the distances between the events estimated using teleseismic $\mathrm{P}$ phases are found to be approximately $25 \%$ shorter than the distances between events estimated using regional Pn phases. The 2009, 2013, and 2016 events all take place within $1 \mathrm{~km}$ of each other and the discrepancy between the regional and teleseismic relative location estimates is no more than about $150 \mathrm{~m}$. The discrepancy is much more significant when estimating the location of the more distant 2006 event relative to the later explosions with regional and teleseismic estimates varying by many hundreds of meters. The relative location of the 2006 event is challenging given the smaller number of observing stations, the lower signal-to-noise ratio, and significant waveform dissimilarity at some regional 
stations. The 2006 event is however highly significant in constraining the absolute locations in the terrain at the Punggye-ri test-site in relation to observed surface infrastructure. For each seismic arrival used to estimate the relative locations, we define a slowness scaling factor which multiplies the gradient of seismic traveltime versus distance, evaluated at the source, relative to the applied 1-d velocity model. A procedure for estimating correction terms which reduce the double-difference time residual vector norms is presented together with a discussion of the associated uncertainty. The modified velocity gradients reduce the residuals, the relative location uncertainties, and the sensitivity to the combination of stations used. The traveltime gradients appear to be overestimated for the regional phases, and teleseismic relative location estimates are likely to be more accurate despite an apparent lower precision. Calibrations for regional phases are essential given that smaller magnitude events are likely not to be recorded teleseismically. We discuss the implications for the absolute event locations. Placing the 2006 event under a local maximum of overburden at $41.293^{\circ} \mathrm{N}, 129.105^{\circ} \mathrm{E}$ would imply a location of $41.299^{\circ} \mathrm{N}$, $129.075^{\circ} \mathrm{E}$ for the January 2016 event, providing almost optimal overburden for the later four events.

\section{INTRODUCTION}

Between October 2006 and September 2016, the Democratic People's Republic of Korea (the DPRK or North Korea) conducted five declared underground nuclear tests. All of the events generated seismic waves that were recorded both at regional and teleseismic distances and all took place within a few $\mathrm{km}$ of each other at the Punggye-ri test-site $\left(41.29^{\circ} \mathrm{N}, 129.10^{\circ} \mathrm{E}\right)$ in the mountainous North of the country. The dates and times of these five events are provided in Tab. 1 together with the globally estimated body-wave magnitudes. These estimates are taken from the Reviewed Event Bulletin (REB) of the International Data Center (IDC) of the Comprehensive Nuclear-Test-Ban Treaty Organization (CTBTO). The corresponding event location estimates, based on International Monitoring System (IMS) data alone, all fall within a few km of the ground infrastructure visible on Google Earth at $41.279^{\circ} \mathrm{N}, 129.087^{\circ} \mathrm{E}$ which is assumed to be related to a weapons testing facility. This infrastructure falls well within the $1000 \mathrm{~km}^{2}$ region surrounding the seismic event location estimate which would be permissible for an on-site inspection (OSI) following entry into force of the Comprehensive NuclearTest-Ban Treaty (CTBT). While the network event location estimates are good, the similarity of the 
seismic waves generated by one event to those generated by another has implications which are useful for providing additional constraints on the sources.

Firstly, significant waveform similarity at a single station implies that a matched filter or correlation detector can be used to detect subsequent events in the immediate vicinity with a very low detection threshold and a very low false alarm rate (e.g. Gibbons \& Ringdal 2012; Ford \& Walter 2015). The stringent condition of near co-location for this form of detector may be mitigated somewhat by using an empirical subspace detector (Barrett \& Beroza 2014) or, for multiple stations, a region of possible source locations can be scanned for optimal network correlation (the so-called "Match and Locate" procedure: Zhang \& Wen 2015). With sufficient waveform similarity, a direct scaling between aligned traces can result in a more accurate estimate of the relative magnitudes (and yields) of the events than a simple comparison of waveform amplitudes (e.g. Gibbons \& Ringdal 2006). In this paper, we focus upon the scanning of relative source location hypotheses in order to estimate the geometry of the explosion sources at the test site. We apply the so-called double-difference techniques (e.g. Waldhauser \& Ellsworth 2000; Richards et al. 2006) which find the most probable relative event locations by minimizing the residuals between predicted and measured traveltime differences.

Two independent relative location studies were published shortly after the 25 May 2009 nuclear test, both indicating that the 2009 test had taken place approximately $2 \mathrm{~km}$ West North West of the 2006 test. The two studies used mutually exclusive sets of observations with Wen \& Long (2010) exploiting only regional Pn phases (observed on stations at distances within $1500 \mathrm{~km}$ of the source) and Selby (2010) exploiting only teleseismic P (observed at several thousand km). The two results are qualitatively identical with Selby (2010) pointing out that the regional estimate of Wen \& Long (2010) falls within the uncertainty ellipse associated with the teleseismic estimate. However, with the goal of placing the events within the terrain and relative to the test-site infrastructure, the quantitative differences between the two studies are significant with the distance between the two events estimated around 25\% further apart when the regional phases are used. As Selby (2010) points out, the accuracy of measurement is a significant factor. (Measuring the time-delays from array-stacks of correlation functions rather than on single-trace correlation functions was demonstrated to result in a quantifiable reduction in the uncertainty ellipse.) Could measurement uncertainty account for the entire discrepancy or are there deterministic reasons that could be quantified and possibly mitigated?

Fig. 1 shows the geometries of the networks used by Wen \& Long (2010) and Selby (2010) together with calculations of the location of the 2006 event relative to the 2009 event using the time measurements provided in these publications. The estimates displayed in panels (b) and (d) of Fig. 1 are calculated using the same code and with the ak135 1-dimensional velocity model (Kennett et al. 1995). The procedure is described in more detail in Section 3 and both estimates are consistent with 
the relative location vectors quoted by the authors of these studies. For the regional estimate (Fig. 1 b) the residual norm increases rapidly as our 2006 epicenter hypothesis moves away from the optimal relative location (labelled WL). The orientation of the residual norm ellipse is a function of the geometry of the observing network. The increase in the residual norm for the teleseismic estimate (Fig. 1 d) is less rapid, giving the impression of a lower resolution estimate. The difference in the apparent resolution is a result of the slowness (or ray-parameter) of the waves recorded at the different stations. The regional phases which have propagated only through the crust and uppermost mantle are assumed to have left the test-site at a shallow angle of incidence. The teleseismic phases propagate more steeply down into the mantle such that the slowness is smaller and the apparent velocity, the distance travelled over the ground per unit time, is greater.

Zhang \& Wen (2013) used a different set of stations at regional distances to locate the February 2013 test relative to the 2009 test, finding 2013 located to the South West of 2009 at a distance of approximately $570 \mathrm{~m}$. Gibbons (2013) performed a similar calculation using only stations of the IMS for the CTBT verification regime, predominantly at teleseismic distances. The result obtained here was qualitatively the same but the distance indicated was less than $500 \mathrm{~m}$. There were more sets of observations for the event pair 2009-2013 than for the event pair 2006-2009 for two main reasons. Firstly, more stations had become operational and, secondly, given the larger magnitudes of the events, the signal-to-noise ratio (SNR) was sufficiently high for several stations on which the 2006 signal had an unacceptably low SNR. Given three pairs of events, there is now considerable redundancy in the data with far more observations available than are strictly necessary to perform a relative event location. (This data redundancy was exploited by Murphy et al. 2013, who performed a jack-knife procedure to obtain a set of relative event location estimates that were less dependent upon individual measurements.) By carefully selecting subsets of observations (e.g. all regional or all teleseismic) we can confirm that the use of regional signals in the relative event location estimates leads consistently to inter-event distances which are greater than the corresponding estimates made using teleseismic signals. This is to say that the discrepancy observed in Fig. 1 is not simply due to measurement inaccuracy, or erroneous data (such as a timing error).

A fourth declared DPRK nuclear test took place on January 6, 2016. While with a slightly smaller seismic magnitude than the February 2013 explosion, the event was significantly larger than 2006 or 2009 and was well-recorded both regionally and teleseismically. It took place several hundred meters to the North West of the 2009 and 2013 tests, but presumably in the same mountain and with a greater overburden. A recent study using exclusively data from stations at regional distances (Zhao et al. 2016) placed the January 2016 explosion approximately $500 \mathrm{~m}$ to the West and $900 \mathrm{~m}$ to to the North of the 2013 test. As discussed by Coblentz \& Pabian (2015), it is most likely geologic considerations which 
have resulted in the more recent tests having taken place in a different body of rock to that in which the 2006 test was carried out. There is no visible infrastructure close to the tunnel assumed to have provided access for the 2006 test, suggesting that this part of the test site has been abandoned.

It is our aim to address the differences between the relative event locations for the DPRK nuclear tests obtained using regional and teleseismic data. The obvious reason to do this is to reduce the uncertainty in the event location estimates in order to gain a better overview of the infrastructure at the test-site. However, given that the relative location estimates are fundamentally dependent upon the set of stations used to make the observations, it is clear that bias exists in the estimates. This bias needs to be understood and, if possible, corrected. If a subsequent test significantly smaller than the 2006 event were to be conducted then our seismic observations would almost exclusively be at regional distances; the SNR at teleseismic distances would be too small. We need relative location estimates that are as independent as possible of the set of observations used. The use of a 1-dimensional velocity model alone is clearly insufficient to explain both regional and global observations and some degree of deviation from a layered model is needed.

We explore the extent to which it is possible to modify our understanding of how the seismic wavefield radiates out from Punggye-ri such that a self-consistent set of location hypotheses for the events can be found which gives a satisfactory fit to all the available seismic recordings. We start by providing an overview of the issues involved in the estimation of relative event locations using "precision seismology", particularly with reference to the DPRK test-site. Subsequently, we discuss a procedure for formulating and solving for a small modification to the layered model which may allow us to provide more robust, consistent and plausible relative event location estimates. All timedelay measurements calculated from cross-correlation of the original waveform data are provided in the supplementary information. The implications for the relative and absolute locations of the DPRK nuclear tests taking into account the modifications made are discussed.

This manuscript was first submitted on June 29, 2016, at which time only four declared DPRK nuclear tests had taken place. The fifth test took place on September 9, 2016, and measurements taken from the seismic signals generated by this event were not used in the calculation of correction terms for relative event location. We however conclude this final version of the paper by presenting a location estimate for the September 2016 event relative to the 2009, 2013, and January 2016 tests. 
Table 1. Dates, times and magnitudes of the North Korean nuclear tests in 2006, 2009, 2013, and 2016. Origin times and magnitudes are taken from the Reviewed Event Bulletin (REB) of the International Data Center (IDC) of the Comprehensive Nuclear-Test-Ban Treaty Organization (CTBTO).

\begin{tabular}{lrr}
\hline Date & Origin time & $m_{\mathrm{b}}$ magnitude \\
\hline October 9, 2006 & 2006-282:01.35.27.58 & 4.1 \\
May 25, 2009 & 2009-145:00.54.42.80 & 4.5 \\
February 12, 2013 & $2013-043: 02.57 .50 .80$ & 4.9 \\
January 6, 2016 & $2016-006: 01.30 .00 .49$ & 4.8 \\
September 9, 2016 & $2016-253: 00.30 .00 .87$ & 5.1 \\
\hline
\end{tabular}

\section{ISSUES IN SEISMIC RELATIVE EVENT LOCATION}

The requirements that typically apply to network earthquake location also apply to estimating relative event locations. We need a sufficient number of stations (e.g. Selby 2010) and their azimuthal distribution must be sufficient to constrain the source location in all directions (examine the shapes of the ellipses in Fig. 1 - some stations are far more important than others, indicating a less-than-ideal azimuthal coverage). Fig. 2 displays short waveform segments surrounding the initial P-wave arrival from the first four declared North Korean nuclear tests at four different IMS stations. Each of these panels illustrates one of the fundamental issues associated with the ability to perform relative event location. In Fig. 2 a) we see the Pn arrival, followed by a presumed Pg phase, on a single channel of the USRK array in the Russian Federation at a distance of approximately $440 \mathrm{~km}$. The signals from the 2009, 2013, and January 2016 events show significant similarity and it is possible to identify repeating features in the first few seconds following the signal onset which can be used to estimate accurate time-delays. The station came online in 2008 and there simply is no waveform available for the 2006 event.

Fig. 2 b) displays a teleseismic P-arrival at a single site of the ASAR array in Australia. Data is present for each of the four events at this station, but the SNR is significantly lower for the 2006 test than for the later events. Being an array station with 19 elements over an aperture of approximately 10 $\mathrm{km}$, the SNR is improved significantly by a beamforming operation. However, the noise for the 2006 test is significant for the problem of discerning the shape of the signal and the time of maximum correlation may have a significant error. The stacking of single-channel correlations (Gibbons \& Ringdal 2006) was confirmed by Selby (2010) to give a more accurate time-delay measurement than a correlation of stack-and-delayed channels. The frequency of the teleseismic signal is significantly lower than for the regional signals displayed in the other three panels. This reduces the time-bandwidth product (TBP) and the significance of the correlation maximum is diminished. 
Fig. 2 c) displays the Pn arrivals from the four events at the MJAR array in Japan. The start of the waveform is coherent from event to event, even in the high frequency band displayed (Gibbons \& Ringdal 2012). However, incorporating the correlation-determined time delays from this station in the source location scan displayed in Fig. 1 d), under the assumption of a Pn phase using the ak135 model, increases the residual norm significantly and moves the estimated relative event location. Assuming that no clock-error was present on the instruments (of which there is no evidence for this station) we have to examine the accuracy of the time-delays predicted by our velocity model. The doubledifference approach means that it is not the absolute traveltime that is significant, but the rate at which this traveltime increases with increasing distance from the station, evaluated at the test-site. Addressing this issue is the primary aim of this paper.

Finally, Fig. 2 d) displays Pn (and in principle Pg) recorded at the KSRS array (Wonju) in South Korea at approximately $440 \mathrm{~km}$. These high frequency regional signals have high SNR for all four events. Examining the first few seconds of this arrival, while there are similarities in the waveforms, the signal from 2006 has a fundamentally different shape to the signals from the other tests. Indeed, performing a correlation calculation in the frequency band displayed results in a time-delay estimate which is clearly erroneous. To measure a meaningful time-difference between the four signals we need to consider lower frequencies (e.g. 1-3 Hz), at which the SNR is reduced significantly.

The similarity between the signals from the different tests varies from station to station in a nonintuitive way. The correlation between the 2006 and 2009 signals at MJAR is satisfactory whereas that between the 2006 and 2009 signals at the JNU 3-component station in the south of Japan is not. The HI-NET broadband seismometer network in Japan provides an unprecedented spatial resolution of the wavefield generated by explosions at the test-site. The azimuthal coverage of HI-NET in relation to the source allowed Huang (2008) to calculate an exceptionally accurate source location from simple back-propagation of the signals from over the network. Fig. 3 a) displays the maximum correlation coefficients obtained between 2006 and 2009 signals at the different HI-NET stations. A fixed frequency band (2-8 Hz) and a fixed time-window (20 s) were used to calculate all correlation coefficients displayed. Gibbons \& Ringdal (2012) present a detailed analysis of signals from the 2006 and 2009 tests recorded in Japan, indicating that these parameters provide an optimal Time-Bandwidth Product for examining waveform similarity. It is noted that this extended time-window contains both the direct arrival and a significant length of coda. Note that there is no systematic variation of SNR with location. While a handful of stations with poor correlation (dark blue or green triangles surrounded by yellow/orange/red triangles) are likely to be the result of local noise or interfering signals, most of the stations with poorly correlating signals have high SNR signals for both events. Very high waveform similarity is only observed for a small region of northern Honshu (centered on $38^{\circ} \mathrm{N}, 140^{\circ} \mathrm{E}$ ) with 
waveform similarity diminishing both North and South as we move away from this region. The waveform similarity between 2006 and 2009 on the southern island of Kyushu (on which the IMS station JNU is located) is significantly poorer than on the rest of Japan.

For the 2009 and 2013 events (Fig. 3 b), the waveform similarity is much higher over the whole network. The stations with the poorest waveform correlation are again in the southernmost part of the country although the stations with the greatest waveform similarity are now further south on Honshu (around $36^{\circ} \mathrm{N}, 138^{\circ} \mathrm{E}$ ). HI-NET provides an unparalleled opportunity to examine how the waveform semblance varies with sensor location; the sparser IMS network samples this variable with a coverage too coarse to be able to draw conclusions. (The performance of the matched filter detectors discussed by Gibbons \& Ringdal 2012, may have been considerably poorer if the array had been located on Kyushu.) From the patterns shown in both panels of Fig. 3, we assume that the degree of waveform similarity for high frequency regional signals is determined primarily in the source region, from topography or near-source geological features.

\section{CALCULATING EMPIRICAL SLOWNESS CORRECTIONS}

There are many variations on procedures for estimating relative locations using double-difference type techniques. We follow a procedure similar to that of Selby (2010) except that, by considering only the differences between different observing stations, the origin times of the different events do not need to be solved for. That is to say that an event $a$ is fixed in space at a location $\boldsymbol{r}_{0}$ and we search a grid of candidate locations $\boldsymbol{r}_{k}$ for the location of a second event, $b$. The location vectors consist of latitude, longitude, and depth. We use the letters $i$ and $j$ to refer to station/phase combinations, e.g. $i$ could refer to WRA (station) and P (phase) whereas $j$ could refer to MJAR (station) and Pn (phase). If a given network (c.f. Fig. 1 a, c) has $n$ station/phase combinations then the preferred relative location of event $b$ is the location $\boldsymbol{r}_{k}$ which minimizes a residual of the form

$$
\left[R\left(\boldsymbol{r}_{0}, \boldsymbol{r}_{k}\right)^{a, b}\right]^{2}=\frac{2}{n(n-1)} \sum_{i=1}^{n-1}\left(\sum_{j=i+1}^{n}\left(f_{i j}^{a b}\left(\boldsymbol{r}_{0}, \boldsymbol{r}_{k}\right)\right)^{2}\right) \text {. }
$$

$R\left(\boldsymbol{r}_{0}, \boldsymbol{r}_{k}\right)^{a, b}$ is the so-called L2 residual norm. There are usually advantages of using the so-called L1 norm (Shearer 1997), although the procedure described here to calculate slowness corrections is more tractable using the L2 norm. The actual location calculations, with or without slowness corrections, can be solved using either L1 or L2 norm minimization. The terms $f_{i j}^{a b}\left(\boldsymbol{r}_{0}, \boldsymbol{r}_{k}\right)$ describe how well the predicted time differences for the locations $\boldsymbol{r}_{0}$ and $\boldsymbol{r}_{k}$ match the measurements for the two station/phase combinations $i$ and $j$. 
We use $\left(t^{a}\right)_{i}$ to denote the reference (epoch) time for a signal template for event $a$ for the arrival $i$ and we use $\tau(\boldsymbol{r})_{i}^{a}$ to denote a model-predicted traveltime from an origin $\boldsymbol{r}$ to the arrival $i$. (Here the superscript $a$ will just be used for association; the predicted traveltime is of course not dependent upon which event we are referring to.) The (epoch) time $\left(t^{b}\right)_{i}$ is the time at which the maximum correlation for the phase $i$ occurs for event $b$ with the template starting at time $\left(t^{a}\right)_{i}$. The explicit form for the term $f_{i j}^{a b}\left(\boldsymbol{r}_{0}, \boldsymbol{r}_{k}\right)$ is

$$
\begin{gathered}
{\left[\left(t^{a}\right)_{j}-\left(t^{a}\right)_{i}\right]} \\
f_{i j}^{a b}\left(\boldsymbol{r}_{0}, \boldsymbol{r}_{k}\right)=-\left[\left(t^{b}\right)_{j}-\left(t^{b}\right)_{i}\right] \\
+\left[\left(\tau^{a}\left(\boldsymbol{r}_{0}\right)\right)_{i}-\left(\tau^{b}\left(\boldsymbol{r}_{k}\right)\right)_{i}\right] \\
-\left[\left(\tau^{a}\left(\boldsymbol{r}_{0}\right)\right)_{j}-\left(\tau^{b}\left(\boldsymbol{r}_{k}\right)\right)_{j}\right]
\end{gathered}
$$

where we have grouped together the template starting times for the two phases (event $a$ ), the times of maximum correlation for the same phases (event $b$ ), and the model-predicted traveltimes for the locations of the fixed master event $\left(\boldsymbol{r}_{0}\right)$ and location hypothesis $\boldsymbol{r}_{k}$ for arrivals $i$ and $j$.

The template and correlation times are fixed from the waveform data and can be grouped into a single term

$$
\Theta_{i j}^{a b}=\left(t^{a}\right)_{j}-\left(t^{a}\right)_{i}-\left(t^{b}\right)_{j}+\left(t^{b}\right)_{i}
$$

which is independent of the location hypothesis $\boldsymbol{r}_{k}$. Unlike the correlation calculations displayed in Fig. 2, for which an extended time-window is used, the window length and frequency band used to calculate correlation-based time-delays were chosen on a phase-by-phase basis to include (as far as possible) only the direct arrival. The window is typically of length $2.5-4.0 \mathrm{~s}$ and the frequency band is chosen to provide the optimal trade-off between SNR and waveform coherence. Time-delay measurements in all cases were accepted or rejected on the basis of a manual inspection of the aligned waveforms. A high or low value of the correlation coefficient is usually a good indicator of the quality of the measurement, although the ultimate decision was a human judgement of waveform similarity. While all the time-delays used in this study were calculated using a correlation computation, we note that a careful manual re-alignment of waveforms may allow for accurate time-delay estimates even when the time of maximum correlation indicates an erroneous time-delay (c.f. Fisk 2002).

Similarly, we can simplify the terms with the model-predicted traveltimes by setting

$$
T_{i}^{a b}=\left(\tau^{a}\left(\boldsymbol{r}_{0}\right)\right)_{i}-\left(\tau^{b}\left(\boldsymbol{r}_{k}\right)\right)_{i}
$$

and

$$
T_{j}^{a b}=\left(\tau^{a}\left(\boldsymbol{r}_{0}\right)\right)_{j}-\left(\tau^{b}\left(\boldsymbol{r}_{k}\right)\right)_{j}
$$


where $T_{i}^{a b}$ and $T_{j}^{a b}$ are functions only of the location variables $\boldsymbol{r}_{0}$ and $\boldsymbol{r}_{k}$. Equation 2 can then be rewritten

$$
f_{i j}^{a b}\left(\boldsymbol{r}_{0}, \boldsymbol{r}_{k}\right)=\Theta_{i j}^{a b}+T_{i}^{a b}-T_{j}^{a b}
$$

where $T_{i}^{a b}$ and $T_{j}^{a b}$ are the differences in the traveltimes between the source locations for phases $i$ and $j$. On the assumption of equal depth, these times are directly proportional to the horizontal slownesses $s_{i}$ and $s_{j}$ which are in turn the inverses of the apparent velocities $v_{\text {app }}^{i}$ and $v_{\text {app }}^{j}$, the velocities at which the wavefronts which ultimately are recorded as phases $i$ and $j$ appear to cover the horizontal ground at the test site. The assumption of equal depth is less of a limitation than it may appear. The residual grids (as displayed in Fig. 1) can also be calculated in 3-dimensional volumes and it can be demonstrated that, as in classical earthquake location problems (e.g. Dixit et al. 2015), the residual pattern changes far less with depth than with lateral distance. We observe the events from different directions, and moving an epicenter hypothesis towards one station will reduce the traveltime to that station while increasing the traveltime to a station in the opposite direction. A difference in depth has a much smaller trade-off from one station to another and an increase or decrease in traveltime will be observed on all stations, offset against a change in the event's origin time. Given a good azimuthal station coverage, a test carried out at a slightly different depth is likely to indicate the correct lateral distances from the master event.

The slownesses are functions both of the local wave speed and of the angle at which the wavefront descends. A wavefront diving down almost vertically will have a very small slowness value and will appear to cross the ground very rapidly. A wavefront leaving the test-site to become trapped in the crust will have a shallower angle of descent, a larger slowness value, and will appear to cover the ground less rapidly. The discrepancy between the regional and teleseismic estimates for the relative event locations has to be due to the $T_{i}^{a b}$ and $T_{j}^{a b}$ predicted by the 1-d velocity model not being accurate. We wish to introduce correction factors $\alpha_{i}$ and $\alpha_{j}$ which scale the slownesses and therefore the times $T_{i}^{a b}$ and $T_{j}^{a b}$. These corrections cannot be identical for all phases. If this were the case then the distances between event location estimates would simply scale linearly with the universally applied scaling factor. The expression for the $f_{i j}$ including the (unknown) scaling factors is

$$
f_{i j}^{a b}\left(\boldsymbol{r}_{0}, \boldsymbol{r}_{k}\right)=\Theta_{i j}^{a b}+\alpha_{i} T_{i}^{a b}-\alpha_{j} T_{j}^{a b}
$$

If our velocity model is close to reality then the slowness adjustments, $\alpha$, will be close to unity. If we have a total of $n$ phases, then we have $n$ different values of $\alpha_{i}$ to find. A direct numerical search of all possible values of all $\alpha$ is intractable. Some progress can be made by setting

$$
\alpha_{i}=1
$$


initially for all station/phase combinations $i$ and then performing grid searches holding all $\alpha$ constant except for one and finding the value of that $\alpha_{i}$ which resulted in the lowest minimum. For instance, we can take the set of teleseismic phases used by Selby (2010) together with the Pn phase at MJAR and perform a number of grid searches (c.f. Fig. 1 d), each with a different value of $\alpha$ for MJAR Pn but with all teleseismic $\mathrm{P} \alpha$ held at unity. In this procedure, a value of about 1.25 for $\alpha$ (MJAR,Pn) appears to produce the lowest residual, although the estimates vary somewhat when different event pairs are considered. This form of direct numerical parameter search can use either L1 or L2 norms for the double difference residual vectors.

The direct search procedure is used to provide ballpark estimates for the likely $\alpha$ parameter ranges for different sets of stations. However, it may be sampling a part of the parameter space close to a local minimum which is far from the true solution. It is necessary to try to solve simultaneously for a set of $\alpha$ which minimize the residual in Eq. 1. However, in addition to the unknown relative location estimates, we then consider an additional unknown for each phase used. The problem is also non-linear. For each phase $i$, we have an initial estimate, $\alpha_{i}^{(0)}$, and then proceed iteratively using

$$
\alpha_{i}^{(m)}=\alpha_{i}^{(m-1)}+\delta \alpha_{i}^{(m)}
$$

for what is essentially a Newton-Raphson type process. For a given location hypothesis for event $b$ (i.e. $\boldsymbol{r}_{k}$ ) we wish to try to solve for a set of $\alpha_{i}$ that result in

$$
R\left(\boldsymbol{r}_{0}, \boldsymbol{r}_{k}\right)^{a, b}=0
$$

Each of the squared terms in Eq. 1 can be written

$$
\begin{gathered}
\left(\Theta_{i j}^{a b}\right)^{2} \\
+\left(\alpha_{j}\right)^{2}\left(T_{j}^{a b}\right)^{2} \\
+\left(\alpha_{i}\right)^{2}\left(T_{i}^{a b}\right)^{2} \\
\left.-2 . \Theta_{i j}^{a b} \cdot \alpha_{j} T_{j}^{a b}\left(\boldsymbol{r}_{0}, \boldsymbol{r}_{k}\right)\right]^{2}=\quad \\
+2 . \Theta_{i j}^{a b} \cdot \alpha_{i} T_{i}^{a b} \\
-2 . \alpha_{j} T_{j}^{a b} \cdot \alpha_{i} T_{i}^{a b}
\end{gathered}
$$

and we substitute the $\alpha_{i}$ and $\alpha_{j}$ as prescribed in Eq. 9. For a given iteration $m$, we have the previous iteration's values $\alpha_{i}^{(m-1)}$ and must solve for the modification terms $\delta \alpha_{i}^{(m)}$. All terms which are nonlinear in the $\delta \alpha_{i}^{(m)}$ terms are neglected.

For a single iteration, the linearized form of Eq. 1 can be expressed as

$$
A \boldsymbol{x}=\boldsymbol{d}
$$

where the solution vector $\boldsymbol{x}$ contains the terms $\delta \alpha_{i}^{(m)}$ for each of the $N$ phases available for the given pair of events. Each row, $k$, of the matrix equation 12 corresponds to a carefully chosen combination 
of the available set of stations. Each subset $k$ must have a sufficient number of stations and a sufficient azimuthal coverage to be able to find a well-defined location minimum. Figure 4 displays the set of stations for which waveforms were obtained for the current study with an indication of those stations which are available for all the first four events. For a given pair of events, the full set of observing stations could form one row of the matrix $\boldsymbol{A}$. Those stations at teleseismic distances could form another row, as could the set of stations at regional distances. Other rows could consist of terms from diverse combinations of stations at both regional and teleseismic distances. If the selected number of meaningful combinations of phases is $K$ then the data matrix $\boldsymbol{A}$ has dimensions $K \times N$. It is likely that the number of rows is less than the number of unknowns (i.e. $K<N$ ) in which case a minimum norm solution to the underdetermined system of linear equations is sought. For a given iteration, the $\alpha$ terms are modified using Eq. 9 and the process is deemed to converge if the norm of the solution vector $\boldsymbol{x}$ tends to zero with subsequent iterations.

For iteration $m$ we begin with a zero $K \times N$ data matrix $\boldsymbol{A}$ and a zero vector $\boldsymbol{d}$ of length $K$. For each subset of stations $k$, we consider each phase combination $(i, j$, with $i<j)$ and perform the operations

$$
\begin{gathered}
{[\boldsymbol{A}]_{k i}=[\boldsymbol{A}]_{k i}+\left[\begin{array}{c}
-2 \alpha_{i}^{(m-1)}\left(T_{i}^{a b}\right)^{2} \\
-2 \cdot \Theta_{i j}^{a b} \cdot T_{i}^{a b} \\
+2 \cdot T_{j}^{a b} \cdot T_{i}^{a b} \cdot \alpha_{j}^{(m-1)}
\end{array}\right],} \\
{[\boldsymbol{A}]_{k j}=[\boldsymbol{A}]_{k j}+\left[\begin{array}{c}
-2 \alpha_{j}^{(m-1)}\left(T_{j}^{a b}\right)^{2} \\
+2 \cdot \Theta_{i j}^{a b} \cdot T_{j}^{a b} \\
+2 \cdot T_{j}^{a b} \cdot T_{i}^{a b} \cdot \alpha_{i}^{(m-1)}
\end{array}\right],}
\end{gathered}
$$

and

$$
[\boldsymbol{d}]_{k}=[\boldsymbol{d}]_{k}+\left[\begin{array}{c}
\left(\Theta_{i j}^{a b}\right)^{2} \\
+\left(\alpha_{j}^{(m-1)}\right)^{2}\left(T_{j}^{a b}\right)^{2} \\
+\left(\alpha_{i}^{(m-1)}\right)^{2}\left(T_{i}^{a b}\right)^{2} \\
-2 \cdot \Theta_{i j}^{a b} \cdot T_{j}^{a b} \cdot \alpha_{j}^{(m-1)} \\
+2 \cdot \Theta_{i j}^{a b} \cdot T_{i}^{a b} \cdot \alpha_{i}^{(m-1)} \\
-2 \cdot T_{j}^{a b} \cdot T_{i}^{a b} \cdot \alpha_{i}^{(m-1)} \cdot \alpha_{j}^{(m-1)}
\end{array}\right] .
$$

When all the elements of $\boldsymbol{A}$ and $\boldsymbol{d}$ are filled, we solve the linear system, modify the set of $\alpha$, and repeat.

For any given set of parameters, the iteration procedure described above appears to converge successfully to a set of slowness corrections. It is worth conducting the process over a grid of the most plausible relative location estimates in order to evaluate the sensitivity of the estimates to the exact coordinates specified. (While the terms $\Theta_{i j}^{a b}$ are dependent only on the correlation-based measurements 
on the waveform data, the terms $T_{i}^{a b}$ and $T_{j}^{a b}$ are dependent upon the location hypotheses since these terms involve model-predicted traveltimes.) Different values of $\alpha$ will of course result in slight changes to the preferred relative location estimates. The procedure is non-linear and iterative on many levels and it is clear that a range of values for the various parameters sought are likely to explain the imperfect data similarly well.

Fig. 5 shows (using blue symbols) a selection of the $\alpha$ slowness correction factors solved for the iterative procedure described above for different event pairs, different subsets of stations, and different relative hypocenter hypotheses. The values are separated into regional and teleseismic phases and plotted as a function of the azimuth from the test-site (distance is not indicated). Fig. 5 provides an impression of the uncertainty associated with the individual estimates of the $\alpha$. There are a number of reasons for the spread. The correlation-based time-delay measurement is subject both to noise (c.f. Fig. 1 panel b), and to clear differences in the waveforms (c.f. Fig. 1 panel d). Sometimes a change in the applied frequency band and the selected data window will result in a significant shift in the peak of maximum correlation and the analyst makes a subjective decision as to which measurement (if any) should be applied. (Application of a lower frequency band often improves the apparent similarity of the waveforms but reduces the SNR and the sharpness of the correlation peak.) The inversion procedure described solves for all $\alpha_{i}$ simultaneously. An erroneous measurement surrounding any one waveform will have consequences for all slowness corrections and multiple inversions with different stations removed (i.e. a jack-knife procedure) and different combinations of phases are likely to increase the confidence in the set of slowness corrections obtained.

The ability to solve for a slowness correction will also depend upon the geometry of events relative to the station. Imagine the case for which the model-predicted traveltime to a given station is almost identical to two different event location hypotheses at the test-site. If an erroneous correlation-based time-delay measurement for the two events at that station is not indicative of two equidistant events then an exaggerated associated correction term $(\alpha)$ may result to try to compensate. The patterns of $\alpha$ observed in Fig. 5 appear largely consistent as a function of the direction from the test site (we note that the $\alpha$ are solved for as independent variables with no assumptions of correlation between different stations). The variability of different estimates appears significantly smaller for the regional phases (panel a) than for the teleseismic (panel b). The regional phases are higher frequency, with a higher time-bandwidth product (TBP), and (assuming signal coherence) the correlation-based timedelay is more easily determined. The teleseismic phases are lower frequency (lower TBP) and there is greater uncertainty in the delay-time measurement. The apparent velocity of the teleseismic phases is, at the same time, significantly greater than that for the regional phases. So the regional correction 
terms are estimated using an accurate measurement of a relatively long time delay and the teleseismic correction terms are estimated using a less accurate measurement of a far shorter time delay.

The red symbols in Fig. 5 indicate values of $\alpha$ which were selected from the distribution of individual estimates indicated by the blue symbols. The values applied in the calculations presented here are tabulated in the supplementary information. In almost all cases, the value of $\alpha$ selected to represent a phase was chosen to be the median of the individual estimates from the various inversions. However, in a few cases with only 2 observations, such as PETK or PSARR, a value of $\alpha$ was simply drawn from the distribution of points displayed in Fig. 5 which appeared to fit the trends best. There is clearly subjectivity in such a judgement and we would advocate considering a range of scaling factors and examining the effect that different values have on the outcome of relative location calculations. Subsequent events at the Punggye-ri test-site which generate signals that correlate well with the signals from previous tests will increase greatly the number of available measurements. The uncertainty in each individual measurement is likely to be of the same order as at present, but the increased number of individual measurements (blue symbols) will increase the likelihood that a good value will be chosen for the relocation calculations.

Fig. 6 shows the values of the selected $\alpha$ as a function of station location. For the stations in Japan, the correction terms appear highly correlated between neighbouring sensors. All values are positive, suggesting that the wavefronts leaving the test-site that are ultimately recorded in Japan propagate more slowly over the ground in the source region than is suggested by the ak135 model. Despite the increased variability in the estimates for the teleseismic phases, it appears that apparent velocity in the source region is consistently overestimated for the signals recorded in Australia and somewhat underestimated for the signals that are ultimately recorded in Europe. The greatest uncertainty is associated with the correction terms for the stations in central Asia and in North America.

\section{IMPLICATIONS FOR THE LOCATIONS OF THE DPRK NUCLEAR TESTS}

We wish to apply all of the available time-delay measurements from stations at both regional and teleseismic distances to obtain the most robust, consistent, and most accurate relative locations possible. We begin by fixing the location of the 2009 event in space and attempting to calculate the locations of the 2006, 2013, and January 2016 (labelled 2016J) events relative to this point. For each of the event pairs 2009-2006, 2009-2013, and 2009-2016J, we consider relative locations using three different sets of measurements: regional-only, teleseismic-only, and using both regional and teleseismic. For example, when locating the 2006 event relative to the 2009 event, we use the times listed in Table S3 (of the supplementary information) for the regional-only calculation, the times listed in Table S4 for the teleseismic-only calculation, and the contents of tables S3 and S4 combined for the third calculation. 
For each calculation, a dense grid of hypothetical epicenters is searched (with depth fixed to zero) and the location with the smallest residual declared the most likely relative location.

Fig. 7 a) displays the outcome for each of these calculations when all traveltimes are predicted using the unperturbed ak135 model. For each of the event pairs, the regional-only estimate indicates the greatest distance between events and the teleseismic-only estimate indicates the shortest distance between events. For a given event pair, the estimate involving both regional and teleseismic delay measurements typically finds a minimum somewhere between the regional-only and the teleseismiconly preferred locations. The absolute discrepancy between the regional and teleseismic estimates is smallest for the 2013 event, estimated at 500 meters or less from the 2009 reference event, and is greatest for the more distant 2006 event. For the 2006 event, the all-teleseismic and all-regional estimates in Fig. 7 a) fall very close to the Selby (2010) and Wen \& Long (2010) estimates respectively indicating that the networks and measurements used in those studies are representative of the available stations at these distances.

Fig. 7 b) displays the results of the equivalent calculations when the slowness corrections displayed in Figures 5 and 6 are applied. (The values of $\alpha$ used are also tabulated in Tables S25 and S26 of the supplementary information.) For each of the 2006, 2013, and January 2016 events, the relative locations indicated from the three calculations are far closer to each other than in Fig. 7 a) where the corrections are not applied. For the 2013 and January 2016 events, the location estimates relative to the 2009 event using regional and teleseismic data are almost identical. For the 2006 event, the absolute discrepancy between the regional estimate and the teleseismic estimate has been reduced by a factor of 4 from almost 800 meters to less than 200 meters. While we have not been able to eliminate the discrepancy entirely, it is noted that the absolute residual norm for the 2006 event is reduced significantly when the corrections are applied.

For the reasons discussed in Section 2, the time-delay measurements for event pairs involving the 2006 event are fewer and potentially less accurate than those made between the signals from the three later events. We see from Fig. 7 b) that the relative location estimates for the 2009, 2013, and January 2016 events are now essentially independent of the set of stations used and, for the final calculations, we assume these three events to be fixed relative to each other. We seek to locate the 2006 event using all available time-delay measurements relative to each of the other three events. We selected many diverse subsets of the total number of available phases for each calculation: a procedure with similarities to the jack-knife (remove one station at a time) method employed by Murphy et al. (2013). The measurements available for the relative location of the 2006 event are given in tables S3 and S4 (for the 2009 master event), tables S7 and S8 (for the 2013 master event), and tables S11 and S12 (for the January 2016 master event). With a few exceptions, the available stations are indicated by the black 
symbols in Fig. 4. We selected different combinations of these stations which provided a reasonable balance in the geographical distribution of the phase recordings.

Fig. 8 a) displays relative location estimates for the 2006 event using the uncorrected ak135 model. Each symbol indicates a location estimate obtained from the indicated master event using a unique subset of the available time-delay measurements. The spread of the individual estimates is considerable; a circle with an aperture of 700 meters is required to enclose all of the estimates. Arguably of greater significance is that the clusters of event location estimates appear to be quite sensitive to the master event selected. Fig. 8 b) displays the results from the same calculations performed using the slowness corrections presented. The total spread is now greatly reduced and a circle with a diameter of 270 meters would now enclose the total set of estimates. Of at least as great importance is that the distributions of the event location estimates are essentially identical for the different master events.

The residual maps in the relative location calculations described in Section 3 can be denoted $R(\boldsymbol{r})_{k}$, where the integer $k$ implies an event to be sought (in this case the 2006 test), a set of timedelay measurements $\xi_{k}$, and a fixed master event location $\boldsymbol{r}_{\text {Master }}$. We wish to calculate a relative event location for the 2006 event which is in some way aggregated from all of the different calculations and therefore less susceptible to the effects of individual measurements. One estimate would be a geometric median of the minima from the individual $R(\boldsymbol{r})_{k}$ residual maps. An alternative estimate could be obtained by superposing the residual maps obtained for the different master events and timedelay measurements. Since it is the minimum value of the residual map which indicates the favoured location, a simple averaging of the $R(\boldsymbol{r})_{k}$ grids may be skewed unfavourably by poor estimates (i.e. estimates with high residuals). If we have a total of $K$ individual residual maps for the event with unknown relative location, we can define a primitive aggregate grid function

$$
R(\boldsymbol{r})_{\text {Aggr. }}=\left(\frac{1}{K} \sum_{k=1}^{K} \frac{1}{R(\boldsymbol{r})_{k}+\epsilon}\right)^{-1}
$$

where $\epsilon$ is a small number (e.g. 0.005) chosen to ensure that no grids with very small minimum residuals completely dominate the solution.

The aggregate residual functions for the subsets of time-delay measurements displayed in Fig. 8 a) and b) are displayed in Fig. 8 c) and d) respectively for the uncorrected and corrected ak135 models. The minimum value of this aggregate residual grid is almost 2500 meters East South East of the 2009 event when corrections are not applied, and approximately 1920 meters in a similar direction when the corrections are applied. The minimum residual obtained is far smaller when the corrections are applied and the residual grows far more rapidly with increasing distance from this point.

The 9 September 2016 event was by far the largest of the five declared DPRK nuclear tests (Fig. 9 a) and the seismic signals generated on all stations showed far greater similarity to those from the 2009 , 
Table 2. Approximate relative locations of the declared DPRK tests based upon the calculated slowness corrections. The 2016 January 6 and 2016 September 9 explosions are labelled 2016J and 2016S respectively.

\begin{tabular}{llrrr}
\hline Event 1 & Event 2 & Distance (m) & Bearing (1 to 2) & Bearing (2 to 1) \\
\hline 2006 & 2009 & 1920 & $282^{\circ}$ & $102^{\circ}$ \\
2006 & 2013 & 2180 & $274^{\circ}$ & $94^{\circ}$ \\
2006 & $2016 \mathrm{~J}$ & 2570 & $285^{\circ}$ & $105^{\circ}$ \\
2006 & $2016 \mathrm{~S}$ & 2170 & $288^{\circ}$ & $108^{\circ}$ \\
2009 & 2013 & 380 & $230^{\circ}$ & $50^{\circ}$ \\
2009 & $2016 \mathrm{~J}$ & 660 & $292^{\circ}$ & $112^{\circ}$ \\
2009 & $2016 \mathrm{~S}$ & 360 & $324^{\circ}$ & $144^{\circ}$ \\
2013 & $2016 \mathrm{~J}$ & 580 & $327^{\circ}$ & $147^{\circ}$ \\
2013 & $2016 \mathrm{~S}$ & 540 & $9^{\circ}$ & $188^{\circ}$ \\
$2016 \mathrm{~J}$ & $2016 \mathrm{~S}$ & 404 & $83^{\circ}$ & $263^{\circ}$ \\
\hline
\end{tabular}

2013, and January 2016 events than those from the October 2006 event. Fig. 9 b) displays a location estimate for the September 2016 event relative to the three master events indicated using the slowness corrections and a superposition of residual maps from multiple calculations as displayed in Fig. 8. All calculations use time-difference measurements as provided in Table S27 of the supplementary material. The 2006 event was not used as a master event for this calculation since the uncertainty in the relative location of this event is significant compared with the inter-site distances between the other events. Tab. 2 provides the dimensions and geometry of the relative event location estimates for all five declared tests using the time-delay measurements and slowness corrections provided in the supplementary information. The distance between the 2013 and January 2016 tests indicated by combining regional and teleseismic data is considerably less than the $1030 \mathrm{~m}$ indicated by Zhao et al. (2016).

The absolute locations of the events cannot be constrained to an accuracy better than several kilometers using the seismic data alone. The locations relative to each other are constrained to within an uncertainty of at most a few hundred meters and this provides significant constraints as to where the tests could have been carried out at Punggye-ri (see e.g. Coblentz \& Pabian 2015). Fig. 10 displays one possible emplacement of the relative event location estimates provided in Tab. 2 with respect to the ground infrastructure with the absolute coordinates chosen out of considerations of maximizing overburden for both the 2006 test and the four more recent events. Essentially all lateral shifts of the stencil from this anchoring result in a decrease in overburden. Shorter tunnels would result in a more southerly absolute position, although all such possibilities have considerably less overburden and the 
ground terrain is likely to apply considerable constraints. In particular, the valley to the East of the 2006 location hypothesis in Fig. 10 should almost certainly be avoided, reducing the number of likely solutions. The local maximum of overburden in the Eastern part of the test site closest to the visible infrastructure is found close to $41.293^{\circ} \mathrm{N}, 129.105^{\circ} \mathrm{E}$. Applying the relative dimensions provided in Tab. 2 would imply a location of $41.299^{\circ} \mathrm{N}, 129.075^{\circ} \mathrm{E}$ for the January 2016 test. This also places the four later tests under a maximum of overburden. Ultimately, the absolute locations are likely to be determined with the greatest confidence using remote sensing observation of surface deformation (e.g. Zelinski et al. 2014).

\section{CONCLUSIONS}

We have demonstrated that, under the assumption of a 1-dimensional seismic velocity model, the relative location estimates for the declared nuclear tests at the Punggye-ri test-site in the DPRK are surprisingly sensitive to the set of time-delay measurements applied. Specifically, the use of regional Pn phases (observed at stations closer than $1500 \mathrm{~km}$ ) indicates greater distances between the different events than the use of teleseismic $\mathrm{P}$ phases. Using both regional $\mathrm{Pn}$ and teleseismic $\mathrm{P}$ phases results in relative location estimates that in some sense fall between the regional and teleseismic extremes. It is noted that the azimuthal coverage available using openly available teleseismic data is far better than that available using openly available regional data. We note also that the open regional data (predominantly in Japan) covers a wide range of azimuth without teleseismic coverage. We have formulated a simple modification to the double difference minimization problem with scaling factors that multiply the gradient of traveltime versus distance, relative to the ak135 model, evaluated at the test-site, that are applied for each phase used to estimate the relative locations.

We have presented a procedure for estimating the values of these scaling factors from the different time-delay measurements between the signals generated by the four events observed between October 2006 and January 2016. While the uncertainty in the estimates for the scaling factors is considerable, a consistent picture emerges that the regional phases leave the test site with slower apparent velocities than are predicted by the ak135 model. The uncertainty in the teleseismic scaling factors is greater but the indications are that teleseismic phases observed in Australia cover the ground at the test-site slower than expected from the 1-d model and teleseismic phases observed in Europe cover the ground at the test-site faster than expected.

Applying these scaling factors results in relative event location estimates with smaller time residuals and, more importantly, which are far less sensitive to the set of stations used to make the estimates. The event for which the relative location estimate uncertainty is greatest is the 2006 explosion. This is due to the lower number of observations, the lower signal-to-noise ratio, and the poorer waveform sim- 
ilarity. Using the ak135 model alone, relative location estimates for the 2006 event vary considerably with the choice of master event and the set of observations used. When the scaling factor corrections are applied, the time residuals decrease and the location estimates vary far less with the selection of observations and the choice of master event.

Assuming a constant depth for the five events, these scaling factors can be interpreted as corrections to the horizontal slowness of the wavefronts leaving the DPRK test-site. A scaling factor greater than unity implies a more slowly propagating wavefront, most likely a shallower angle of incidence. The scaling factors found to minimize the residual vector norms for the calculations presented here are well within the range of deviations observed for wavefronts arriving at seismic arrays (e.g. Gibbons et al. 2011). Studies of wavefronts approaching seismic arrays however also raise the issue that a significant deviation in the azimuth is frequently observed: a reminder that the corrections prescribed in this paper are a simplification. The wavefield leaving the test-site will be subject to 3-dimensional heterogeneities. The calculated corrections applied to the traveltime curves are a great-circle approximation and also neglect possible changes over the dimensions of the test-site.

We have demonstrated surprising differences in the waveform similarity between the different tests as a function of sensor location, using the very dense HI-NET deployment in Japan. The region of Japan in which the greatest similarity is observed between the signals from the 2006 and 2009 events is different from the region observing the greatest similarity between the 2009 and 2013 signals. This observation can only be explained by effects in the source region. While we cannot identify which near-source heterogeneities would result in these observations, the fact that the explosions are taking place within steep-sided mountains makes it likely that topographic effects are significant in determining the form of free-surface reflections. The greatest similarity between the signals from the 2006 and 2009 tests is observed is at stations close to the great circle path which contains both the 2006 and 2009 events in the relocations performed here.

The relative location estimates obtained using the calculated scaling factors suggest that, despite the poorer apparent resolution, the relative event location estimates using teleseismic phases (e.g. Selby 2010) are probably more accurate than those obtained using only regional phases (e.g. Wen \& Long 2010). However, in the event of a smaller nuclear test at the Punggye-ri site, the regional observations are likely to be the only ones available and accurate relative locations based only upon regional phases are essential. We have provided all of the time-delay measurements used in the calculations displayed.

\section{ACKNOWLEDGMENTS}

This work was partly supported by the Norwegian Ministry of Foreign Affairs. 
Data from International Monitoring System (IMS) stations was obtained from the International Data Center (IDC) of the Comprehensive Nuclear-Test-Ban Treaty Organization (CTBTO) in Vienna, Austria.

We thank the Kazakhstan National Data Center (http://www.kndc.kz) for non-IMS array data from the Kazakhstan network.

Data from the HI-NET High Sensitivity Seismograph Network, Japan, is available from the National Research Institute for Earth Science and Disaster Prevention (NIED), Japan, at http://www.hinet.bosai.go.jp/ (last referenced March 2016, registration required). We are very grateful to the National Research Institute for Earth Science and Disaster Prevention (NIED), Japan, for access to and permission to use the HI-NET seismic data.

Most maps and figures in this paper are created using GMT software (Wessel \& Smith 1995).

\section{REFERENCES}

Barrett, S. A. \& Beroza, G. C., 2014, An Empirical Approach to Subspace Detection, Seismological Research Letters, 85(3), 594-600.

Coblentz, D. \& Pabian, F., 2015, Revised Geologic Site Characterization of the North Korean Test Site at Punggye-ri, Science \& Global Security, 23(2), 101-120.

Dixit, A. M., Ringler, A. T., Sumy, D. F., Cochran, E. S., Hough, S. E., Martin, S. S., Gibbons, S., Luetgert, J. H., Galetzka, J., Shrestha, S. N., Rajaure, S., \& McNamara, D. E., 2015, Strong-Motion Observations of the $\mathrm{M}=7.8$ Gorkha, Nepal, Earthquake Sequence and Development of the N-SHAKE Strong-Motion Network, Seismological Research Letters, 86(6), 1533-1539.

Fisk, M. D., 2002, Accurate Locations of Nuclear Explosions at the Lop Nor Test Site Using Alignment of Seismograms and IKONOS Satellite Imagery, Bulletin of the Seismological Society of America, 92, 29112925 .

Ford, S. R. \& Walter, W. R., 2015, International Monitoring System Correlation Detection at the North Korean Nuclear Test Site at Punggye-ri with Insights from the Source Physics Experiment, Seismological Research Letters, 86, 1160-1170.

Gibbons, S. J., 2013, Detection and Location of the February 12, 2013, Announced Nuclear Test in North Korea, NORSAR Scientific Report: Semiannual Technical Summary No. 2 - 2012, NORSAR, Kjeller, Norway, pp. 26-35.

Gibbons, S. J. \& Ringdal, F., 2006, The detection of low magnitude seismic events using array-based waveform correlation, Geophys. J. Int., 165, 149-166.

Gibbons, S. J. \& Ringdal, F., 2012, Seismic Monitoring of the North Korea Nuclear Test Site Using a Multichannel Correlation Detector, Geoscience and Remote Sensing, IEEE Transactions on, 50, 1897-1909.

Gibbons, S. J., Schweitzer, J., Ringdal, F., Kværna, T., Mykkeltveit, S., \& Paulsen, B., 2011, Improvements to 
Seismic Monitoring of the European Arctic Using Three-Component Array Processing at SPITS, Bulletin of the Seismological Society of America, 101(6), 2737-2754.

Huang, B.-S., 2008, Tracking the North Korean nuclear explosion of 2006, using seismic data from Japan and satellite data from Taiwan, Physics of the Earth and Planetary Interiors, 167, 34-38.

Kennett, B. L. N., Engdahl, E. R., \& Buland, R., 1995, Constraints on seismic velocities in the Earth from traveltimes, Geophysical Journal International, 122, 108-124.

Murphy, J. R., Stevens, J. L., Kohl, B. C., \& Bennett, T. J., 2013, Advanced Seismic Analyses of the Source Characteristics of the 2006 and 2009 North Korean Nuclear Tests, Bulletin of the Seismological Society of America, 103, 1640-1661.

Richards, P., Waldhauser, F., Schaff, D., \& Kim, W.-Y., 2006, The Applicability of Modern Methods of Earthquake Location, Pure and Applied Geophysics, 163(2), 351-372.

Selby, N. D., 2010, Relative Locations of the October 2006 and May 2009 DPRK Announced Nuclear Tests Using International Monitoring System Seismometer Arrays, Bulletin of the Seismological Society of America, 100, 1779-1784.

Shearer, P. M., 1997, Improving local earthquake locations using the L1 norm and waveform cross correlation: Application to the Whittier Narrows, California, aftershock sequence, J. Geophys. Res., 102(B4), 8269-8283.

Waldhauser, F. \& Ellsworth, W. L., 2000, A Double-Difference Earthquake Location Algorithm: Method and Application to the Northern Hayward Fault, California, Bulletin of the Seismological Society of America, 90(6), 1353-1368.

Wen, L. \& Long, H., 2010, High-precision Location of North Korea's 2009 Nuclear Test, Seismological Research Letters, 81, 26-29.

Wessel, P. \& Smith, W. H. F., 1995, New version of the Generic Mapping Tools, EOS Trans. Am. Geophys. Union, 76, 329.

Zelinski, M. E., Henderson, J., \& Smith, M., 2014, Use of Landsat 5 for Change Detection at 1998 Indian and Pakistani Nuclear Test Sites, IEEE Journal of Selected Topics in Applied Earth Observations and Remote Sensing, 7(8), 3453-3460.

Zhang, M. \& Wen, L., 2013, High-precision location and yield of North Korea's 2013 nuclear test, Geophys. Res. Lett., 40, 2941-2946.

Zhang, M. \& Wen, L., 2015, An effective method for small event detection: match and locate (M\&L), Geophysical Journal International, 200, 1523-1537.

Zhao, L.-F., Xie, X.-B., Wang, W.-M., Hao, J.-L., \& Yao, Z.-X., 2016, Seismological Investigation of the January 6, 2016 North Korean Underground Nuclear Test, Geophysical Journal International. 
a)

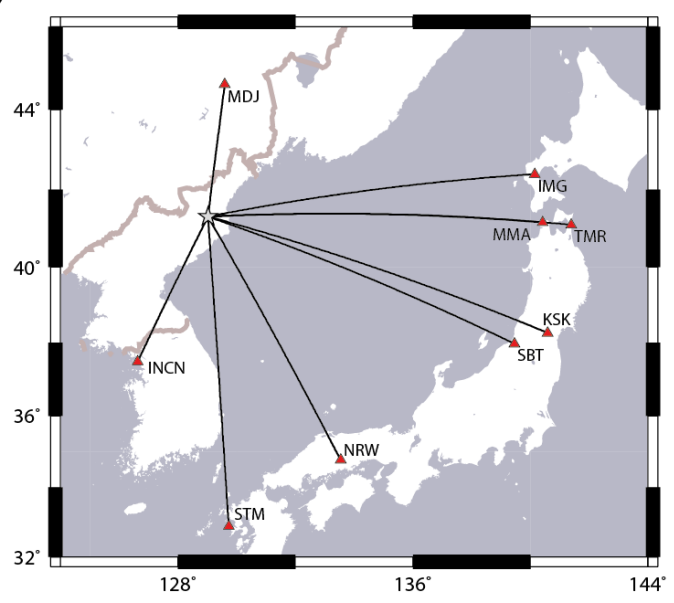

b) $129.05^{\circ}$

$129.10^{\circ}$

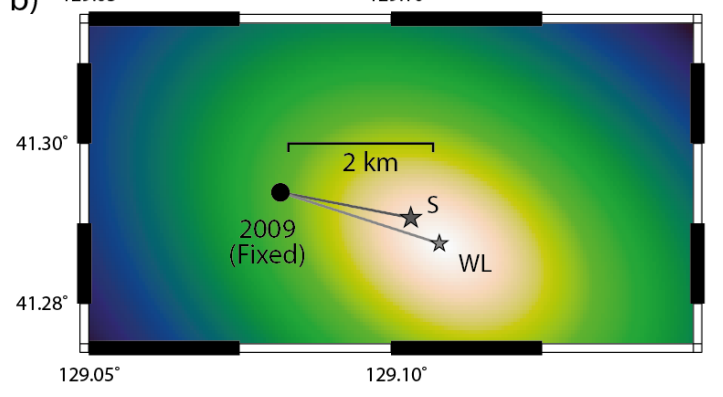

c)

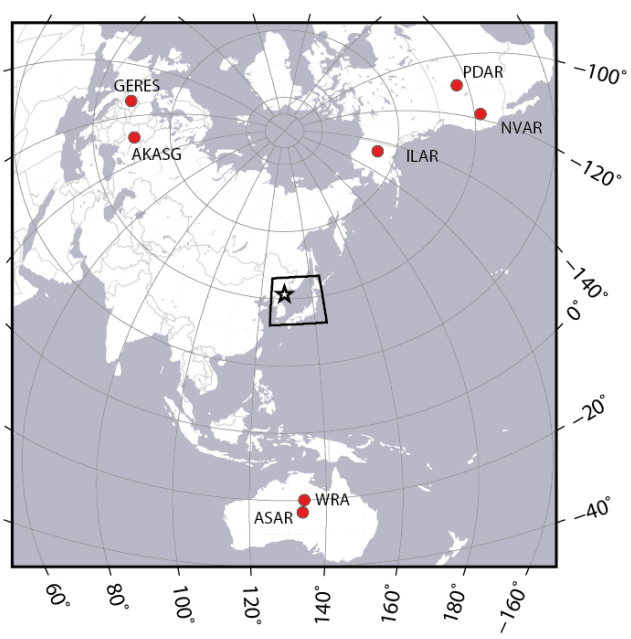

d) $129.05^{\circ}$

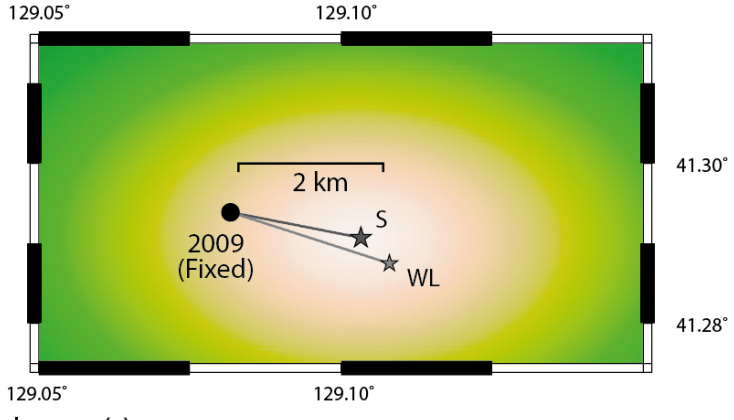

Time residual norm (s)

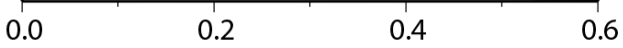

Figure 1. Relative locations of the 2006 and 2009 DPRK nuclear tests using regional and teleseismic data. (a) Network of regional stations used by Wen \& Long (2010) (WL) and (b) residual contours for the 2006 event relative to the 2009 event. (c) Global network of IMS arrays used by Selby (2010) (S) and (d) the corresponding residual contours. The symbols for both relative location estimates are displayed on both residual plots. 
a)

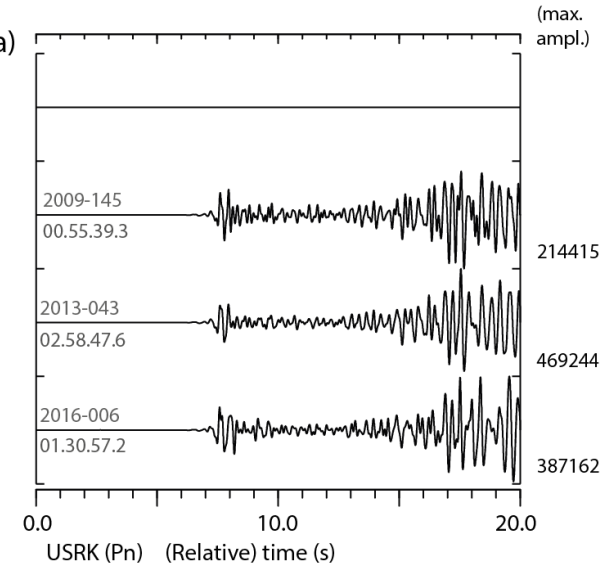

b)

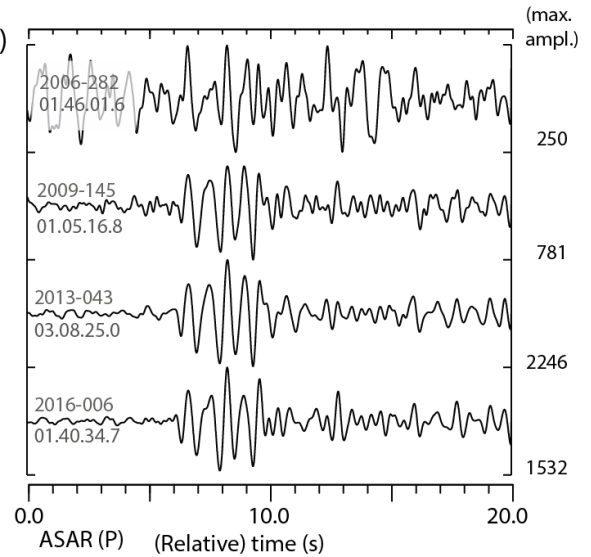

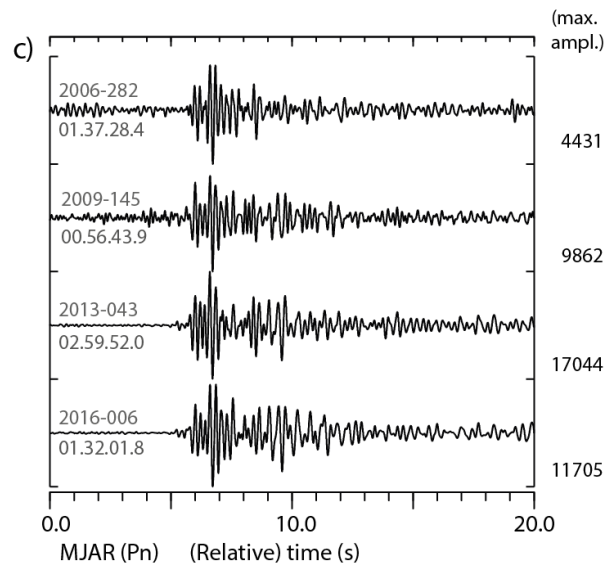

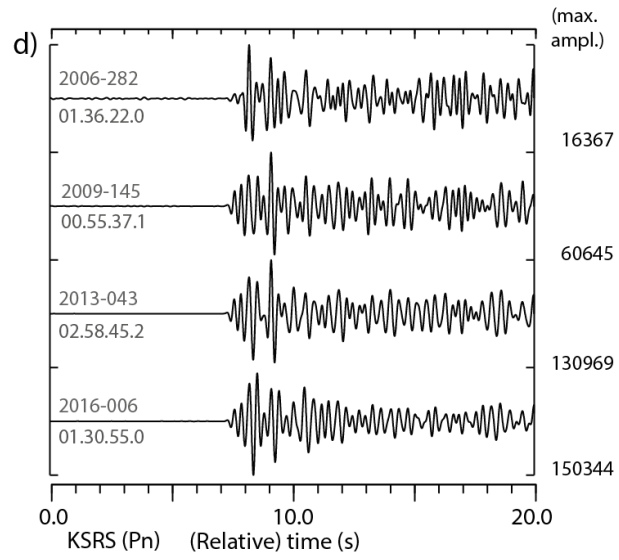

Figure 2. Single vertical channel segments with the first arrivals from the first four DPRK nuclear tests recorded at IMS seismic arrays as displayed. Frequency bands are (a) USRK (2-8 Hz), (b) ASAR (1-4 Hz), (c) MJAR (2-8 $\mathrm{Hz}$ ), and (d) KSRS (2-8 Hz). The starting time of each trace is displayed at left and the maximum amplitude (in counts) at right allows comparison between events. Trace amplitudes are normalized to give unit maximum per channel. The Pn coda for the USRK and KSRS stations is also likely to contain Pg arrivals with a few seconds delay. 
a)

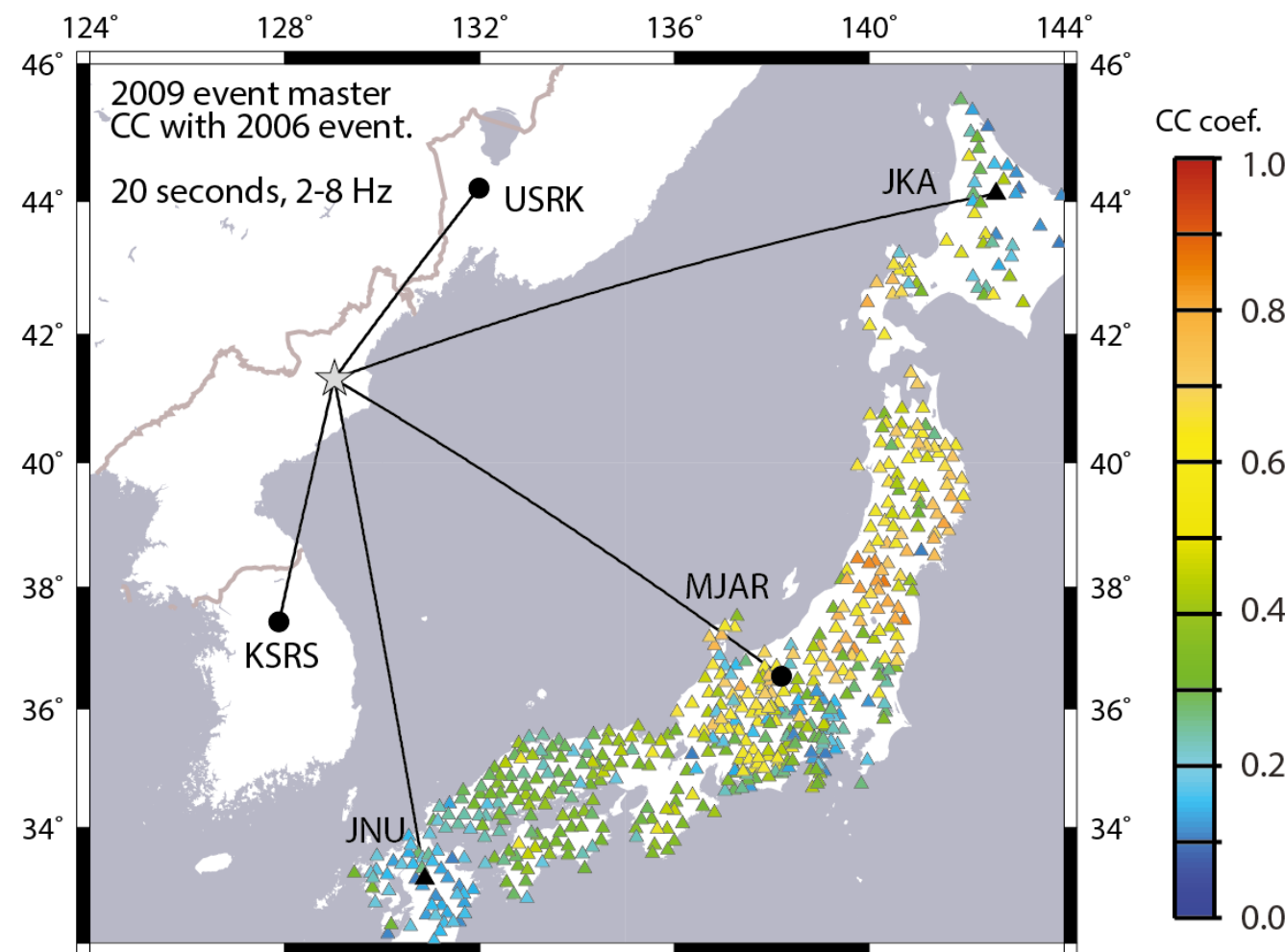

b)

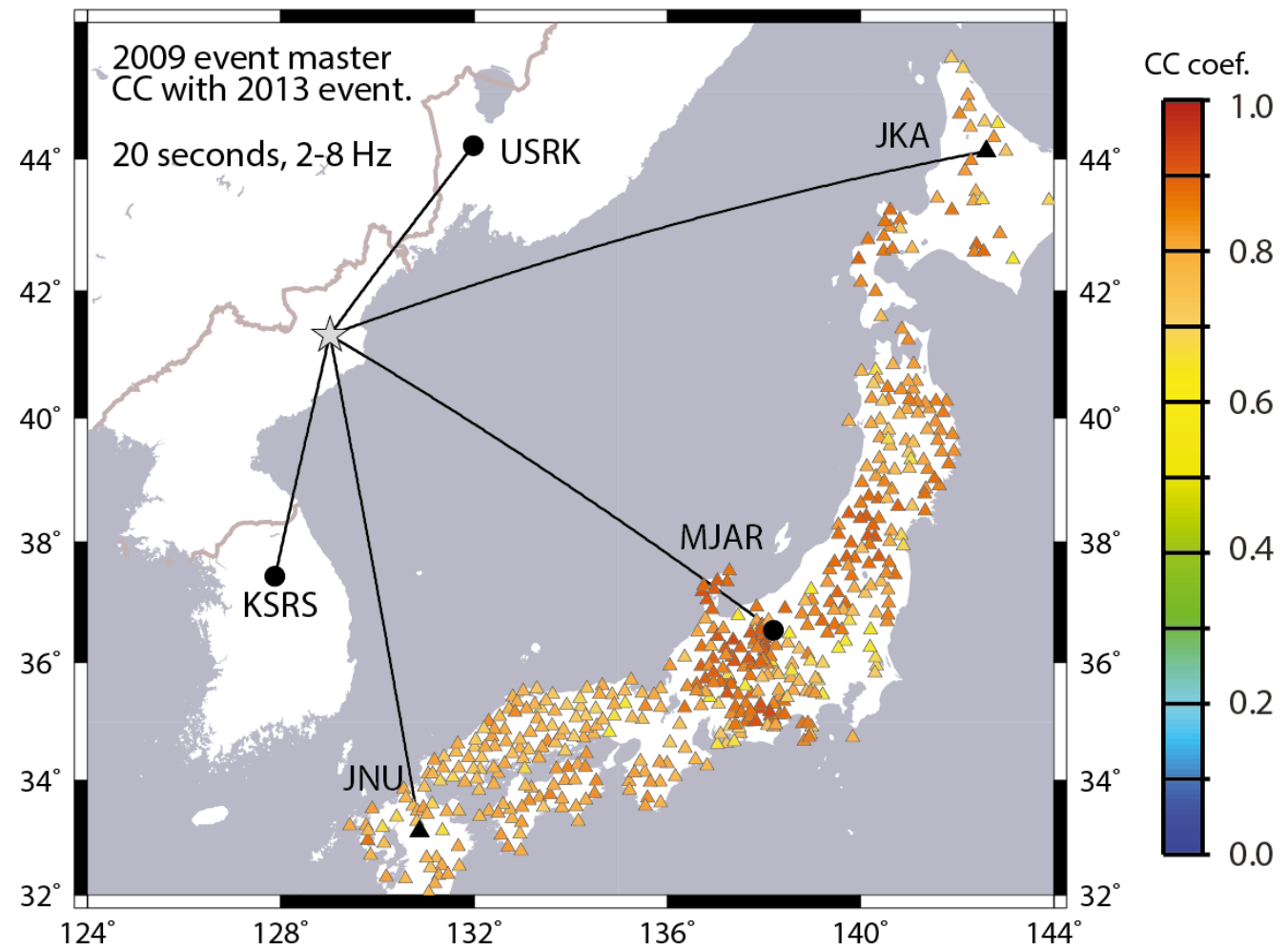

Figure 3. Similarity of waveforms between the 2006 and 2009 events (a) and between the 2009 and 2013 events (b) on stations of the HI-NET network in Japan. The maximum correlation coefficient is measured using all 3-components of each site with a 20 second long waveform template bandpass filtered between 2 and $8 \mathrm{~Hz}$. The black symbols are IMS stations with (from North to South) JKA, MJAR and JNU in Japan, KSRS in South Korea, and USRK in Russia. The star indicates the location of the DPRK test site. 
a)

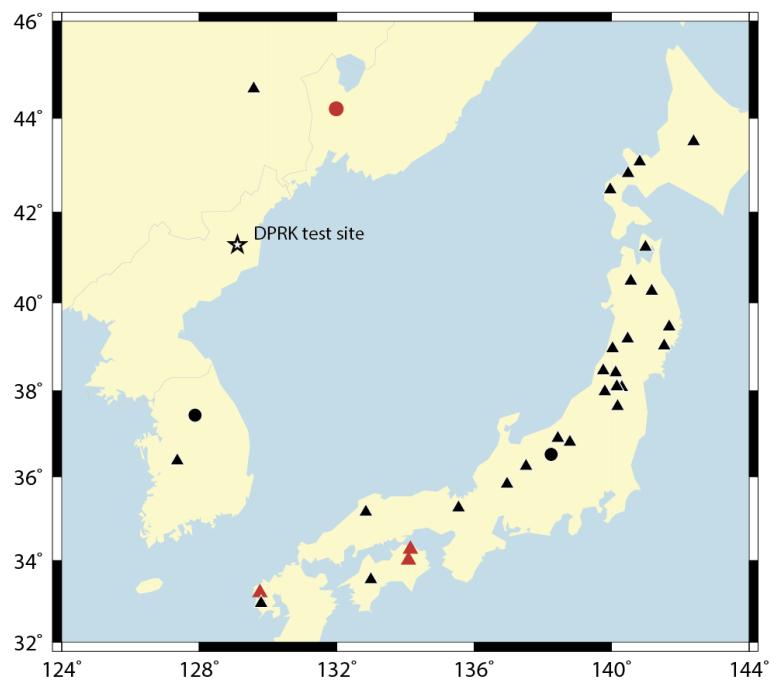

b)

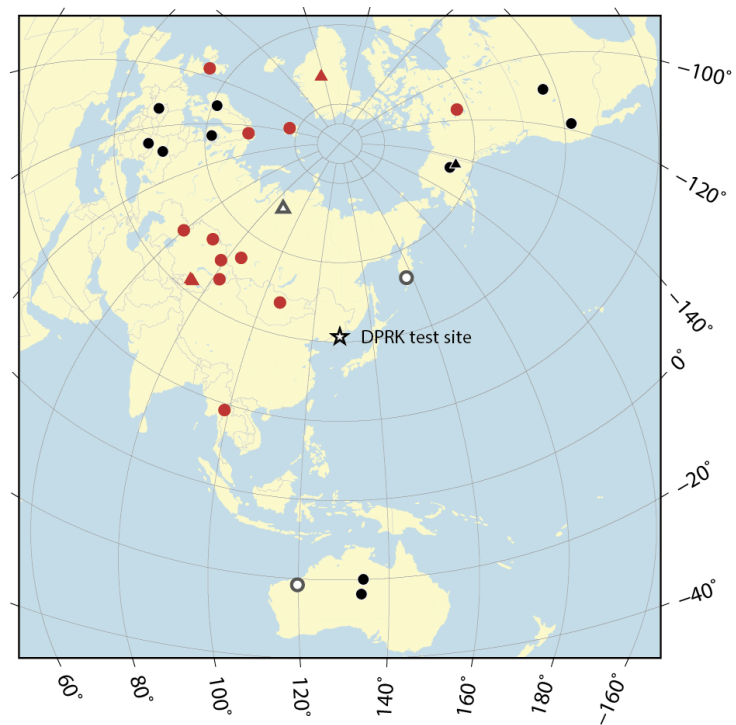

Figure 4. Stations available with satisfactory signal correlations for (a) regional Pn phases and (b) teleseismic P phases for the four DPRK events between October 2006 and January 2016. Arrays and 3-component stations are represented by circles and triangles respectively. Stations with 1,3, and 6 event pairs are marked with white, red, and black symbols respectively. 
a) Regional Pn correction factors

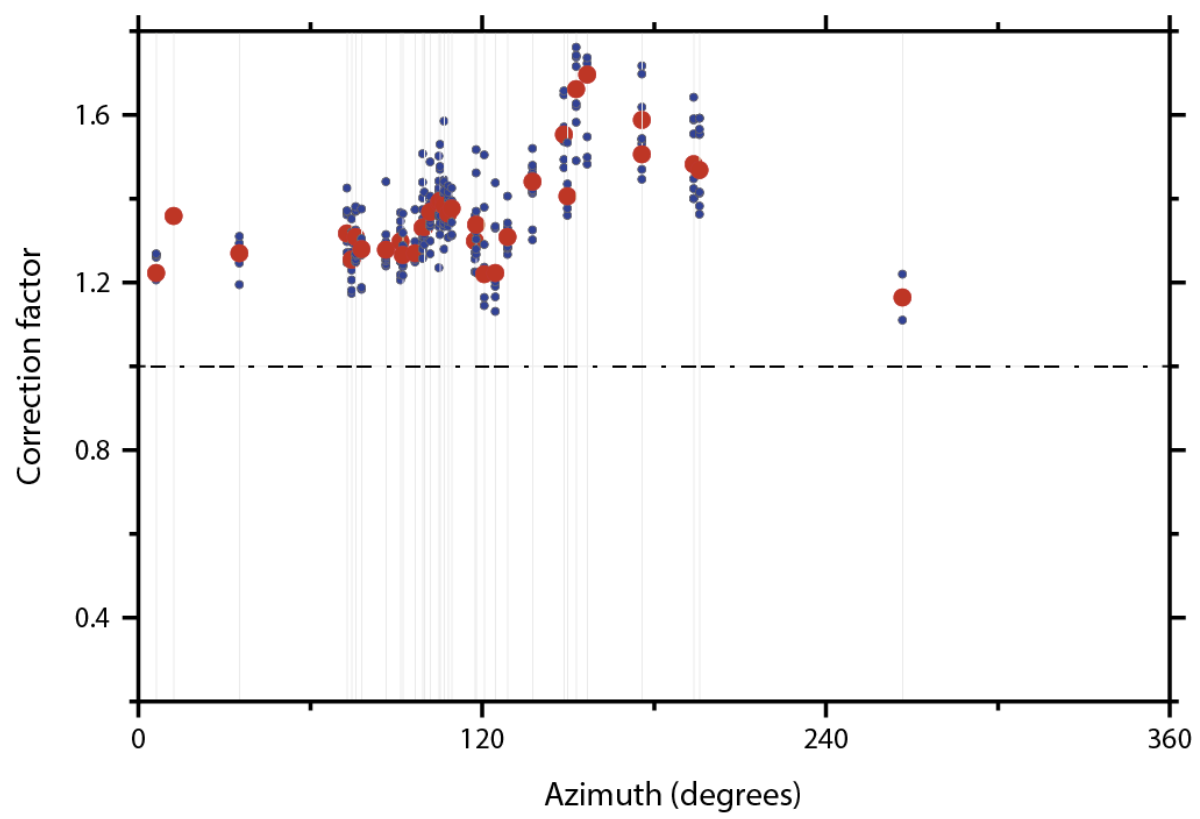

b) Teleseismic P correction factors

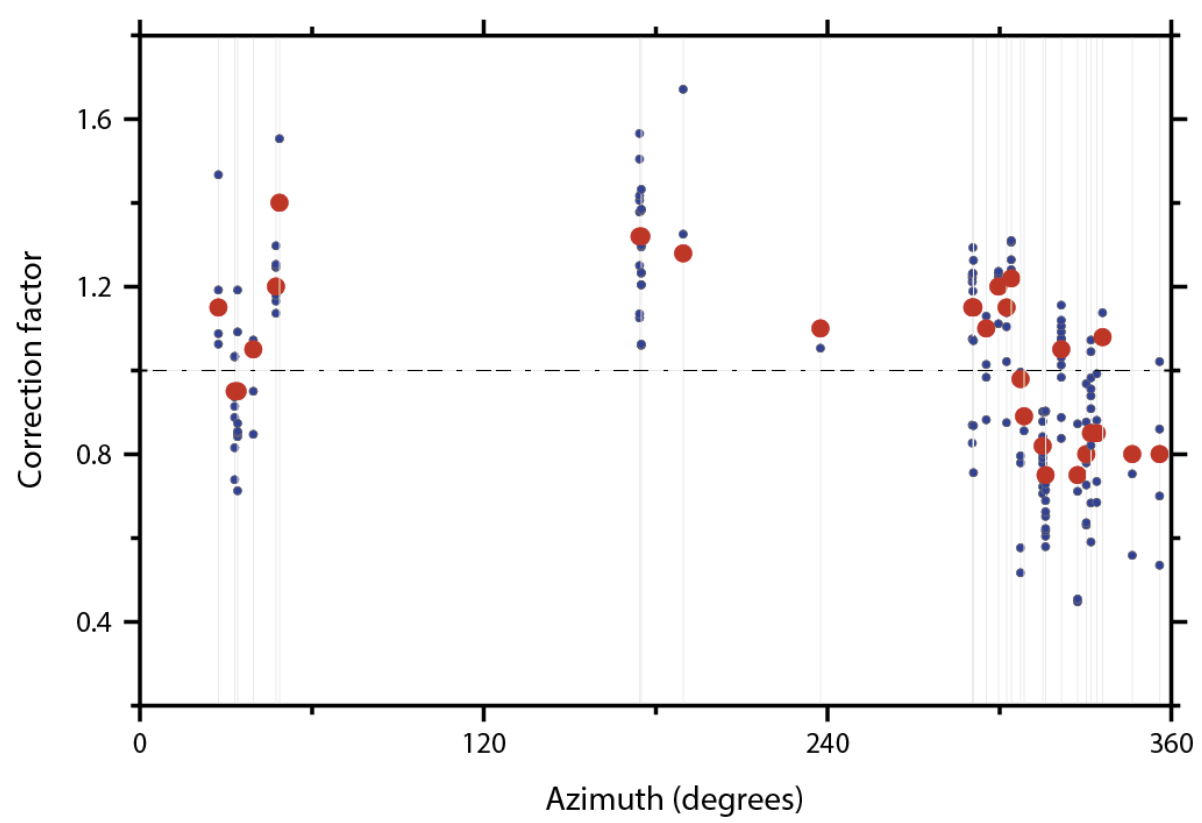

Figure 5. Estimates for slowness correction factors, $\alpha$, for the DPRK test site for a) regional Pn phases and b) teleseismic $\mathrm{P}$ phases as a function of azimuth from the source. The small blue symbols indicate individual estimates for the slowness correction for one particular phase for different sets of time-measurements and/or event location hypotheses. The larger red symbols indicate the $\alpha$ value selected for each phase based upon these distributions (see text). The dashed lines indicate $\alpha=1$ for which the apparent velocity for phases leaving the test site would exactly match those predicted by the ak135 velocity model. 
a)

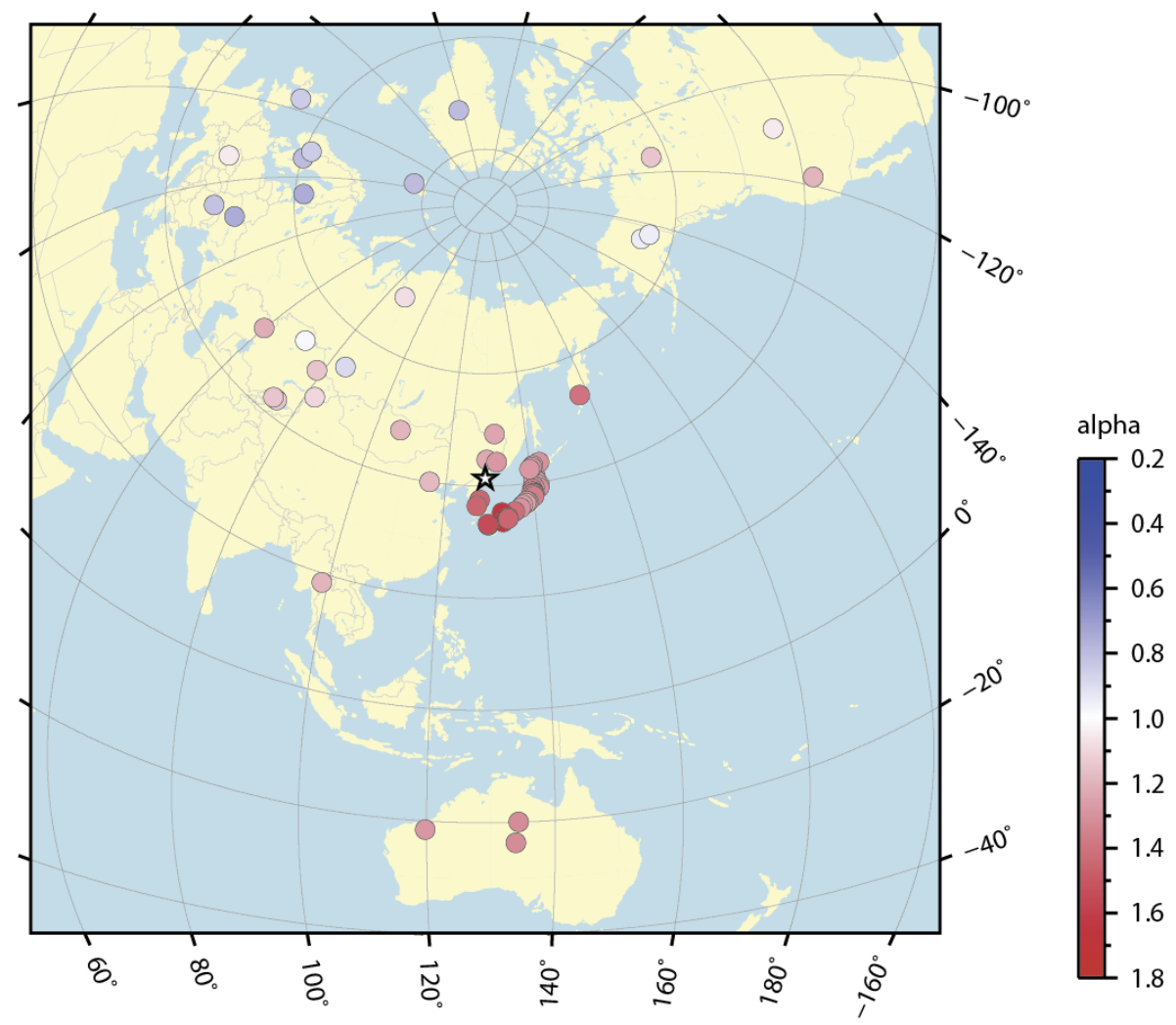

b)

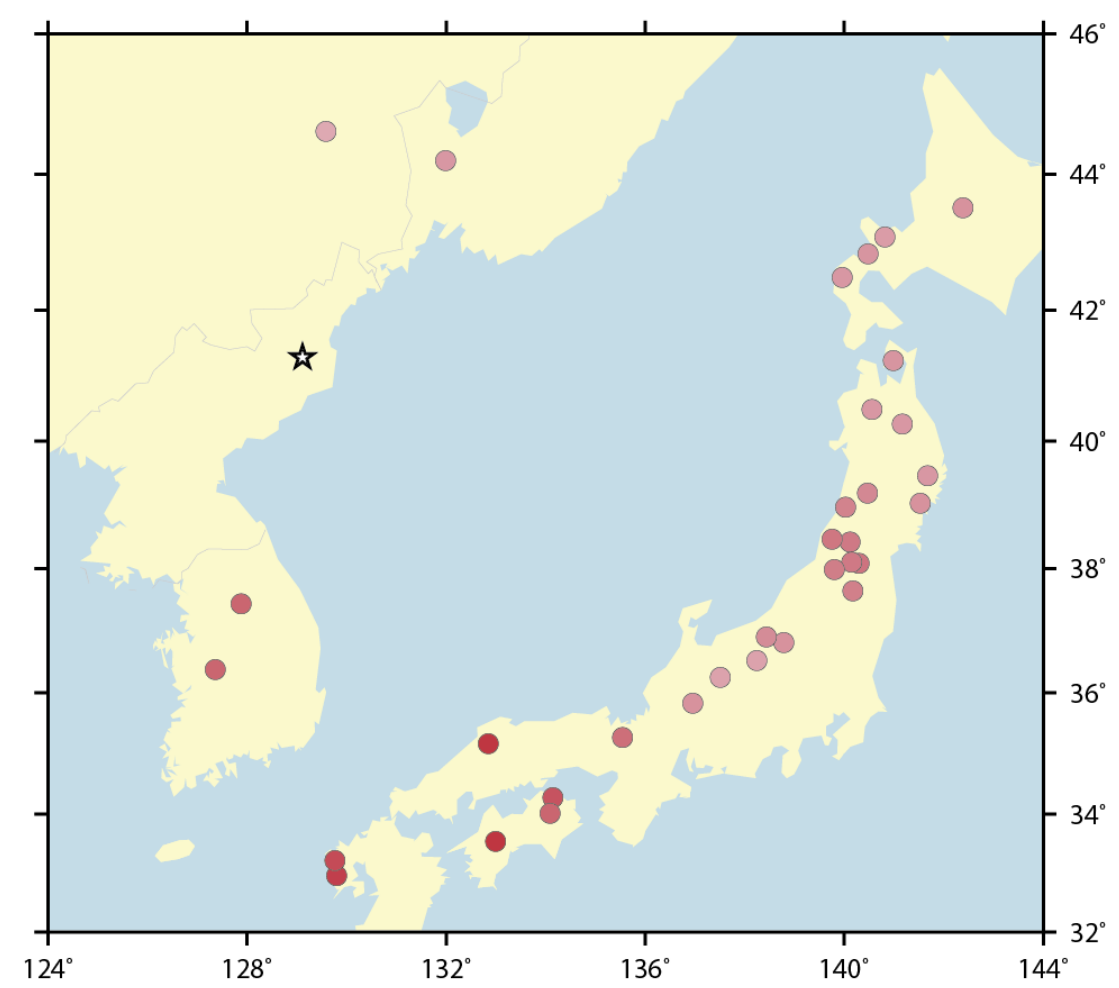

Figure 6. The $\alpha$ selected from Fig. 5 globally (a) and for stations at regional distances (b). The star indicates the location of the DPRK test site. 
a)

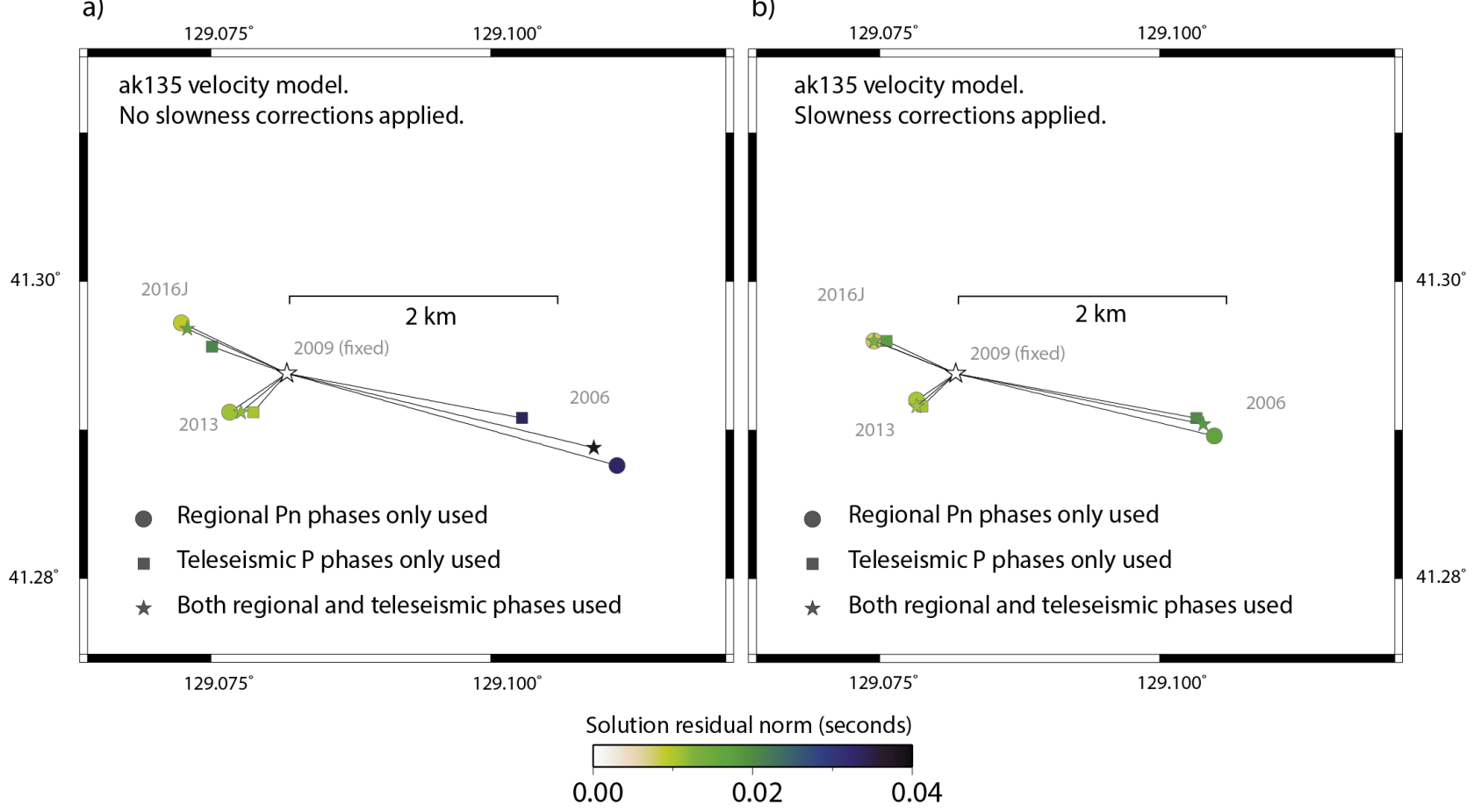

Figure 7. Estimates for the locations of the 2006, 2013, and January 2016 (labelled 2016J) DPRK nuclear tests relative to the (fixed) 2009 event for the indicated combinations of phases (a) for the uncorrected ak135 seismic velocity model and (b) subject to the slowness correction factors $\alpha$ displayed in Fig. 6. The absolute coordinates on the maps are for guidance only; lateral translations of the events are consistent with the seismic data presented in this study. 
a)

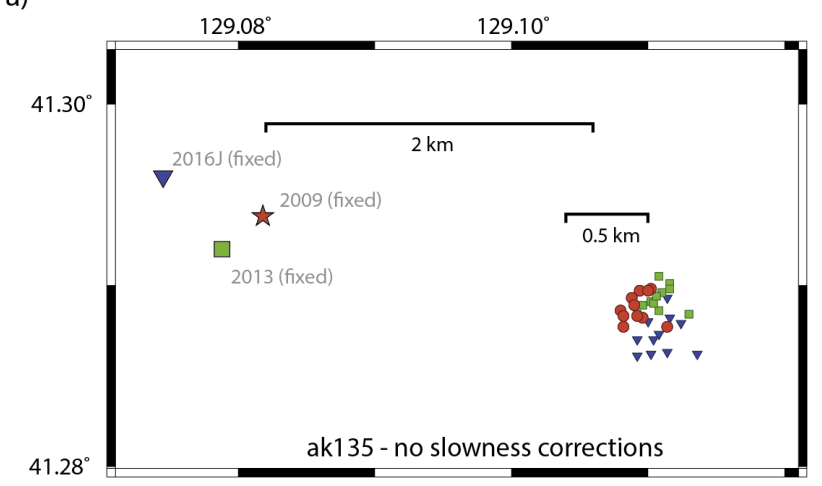

c)

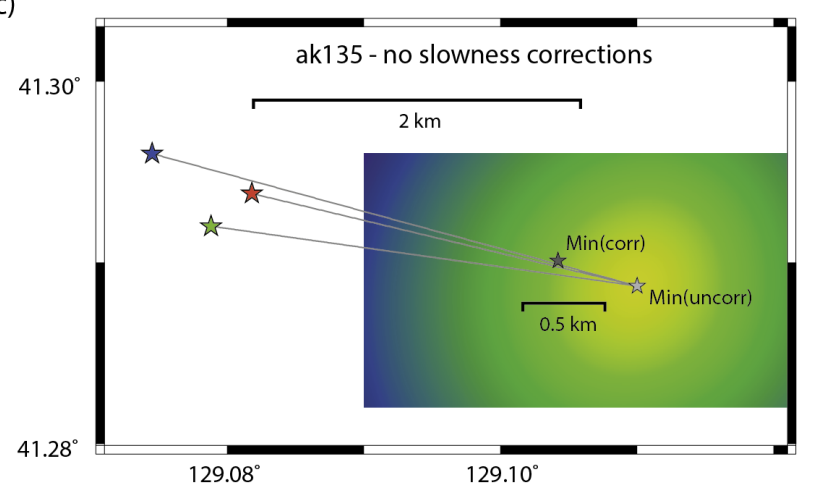

Solution residual norm (seconds)

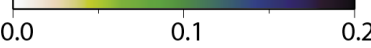

b)

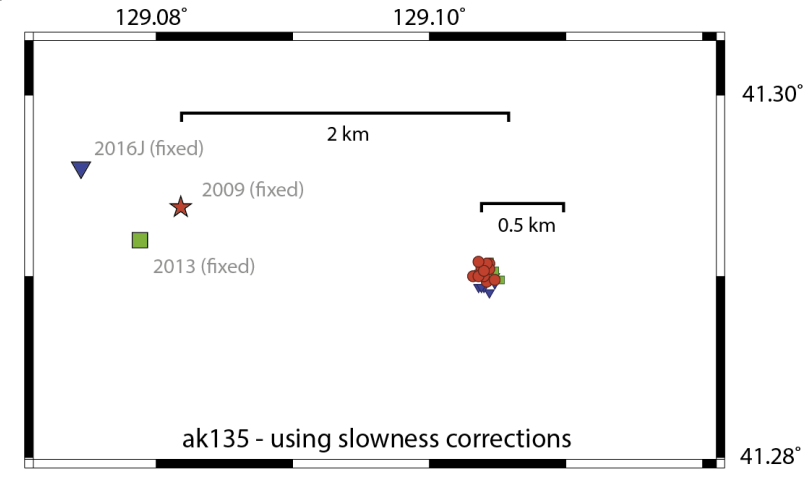

d)

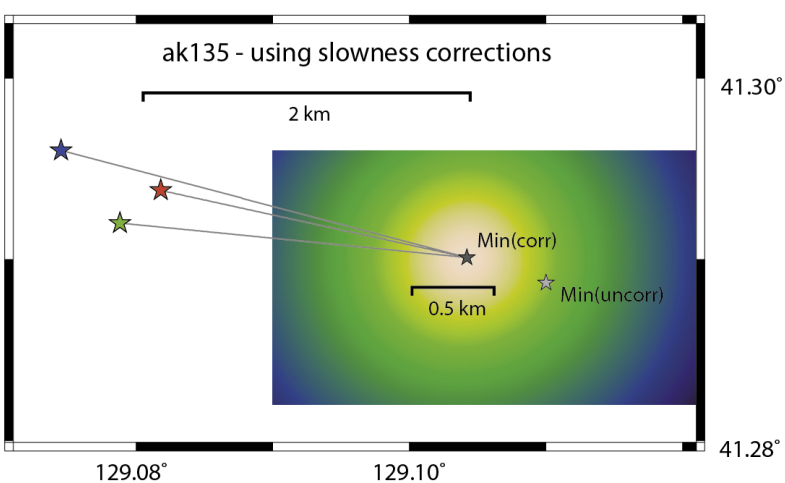

Figure 8. Panels (a) and (b) display relative event location estimates for the 2006 test using different subsets of the available measurements, with and without applied slowness corrections as indicated. The colour and shape of the smaller symbols indicate which of the three fixed master events was used. The subsets contain both regional and teleseismic P phases. Panels (c) and (d) display superpositions of the traveltime difference residual grids calculated for each of the computations performed in panels (a) and (b) respectively. The absolute coordinates on the maps are for guidance only; lateral translations of the events are consistent with the seismic data presented in this study. 


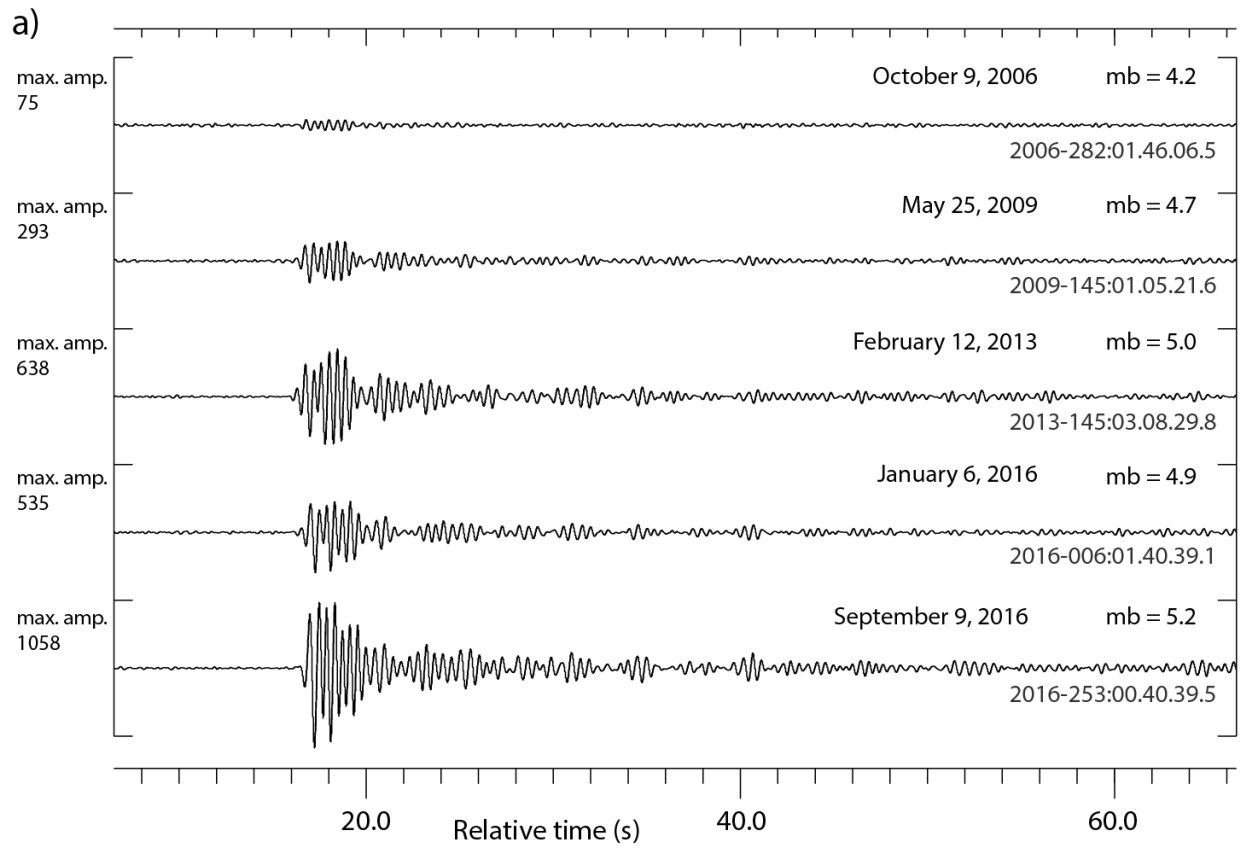

Optimal beam, NORSAR array, Hedmark, Norway (vertical components)

b)

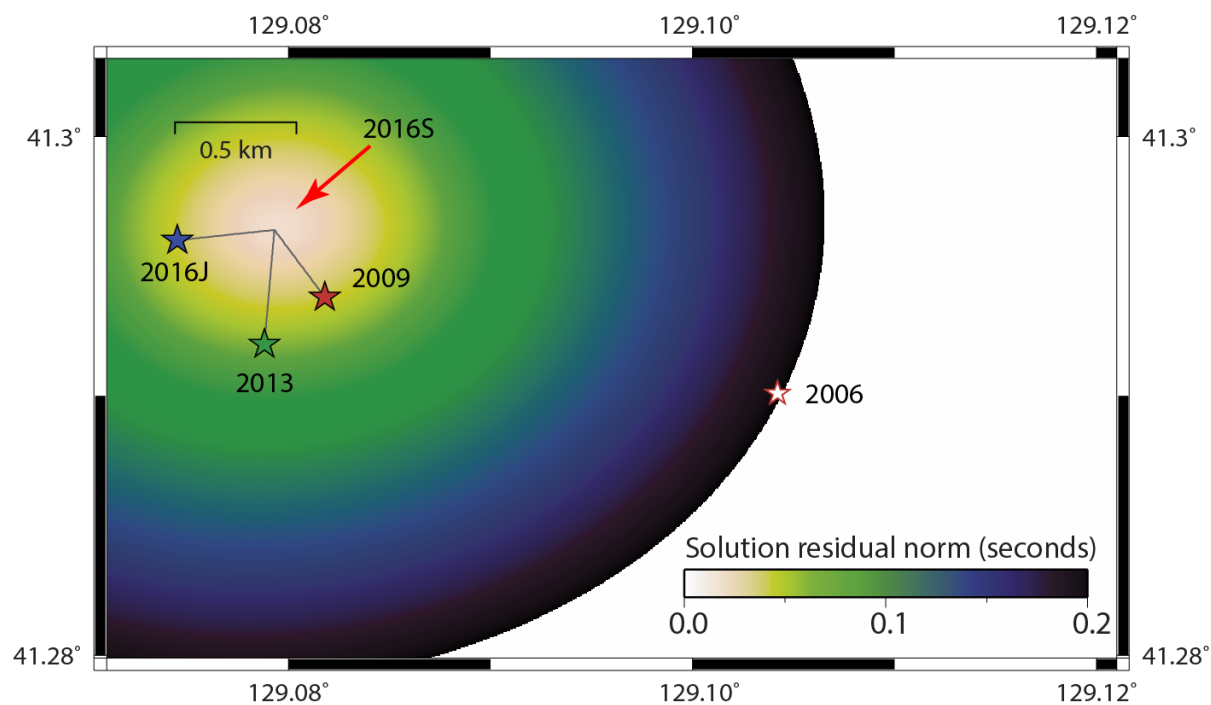

Figure 9. The 9 September 2016 DPRK nuclear test. (a) optimized beams on the NORSAR array, Norway, displayed for each of the five nuclear tests as indicated. All traces shown to the same vertical scale with broadband traces (2013-2016) converted to the short-period instrument response present for the 2006 and 2009 events. The epoch time for the start of each trace is displayed at right and maximum amplitudes are in counts with a 1-5 Hz bandpass filter applied. (b) Relative event location estimate for the 2016 September 9 event (labelled 2009S) constructed as in Fig. 8 from the 2009, 2013 and 2016 January 6 (labelled 2006J) events. The absolute coordinates on the map are for guidance only; lateral translations of the events are consistent with the seismic data presented in this study. 


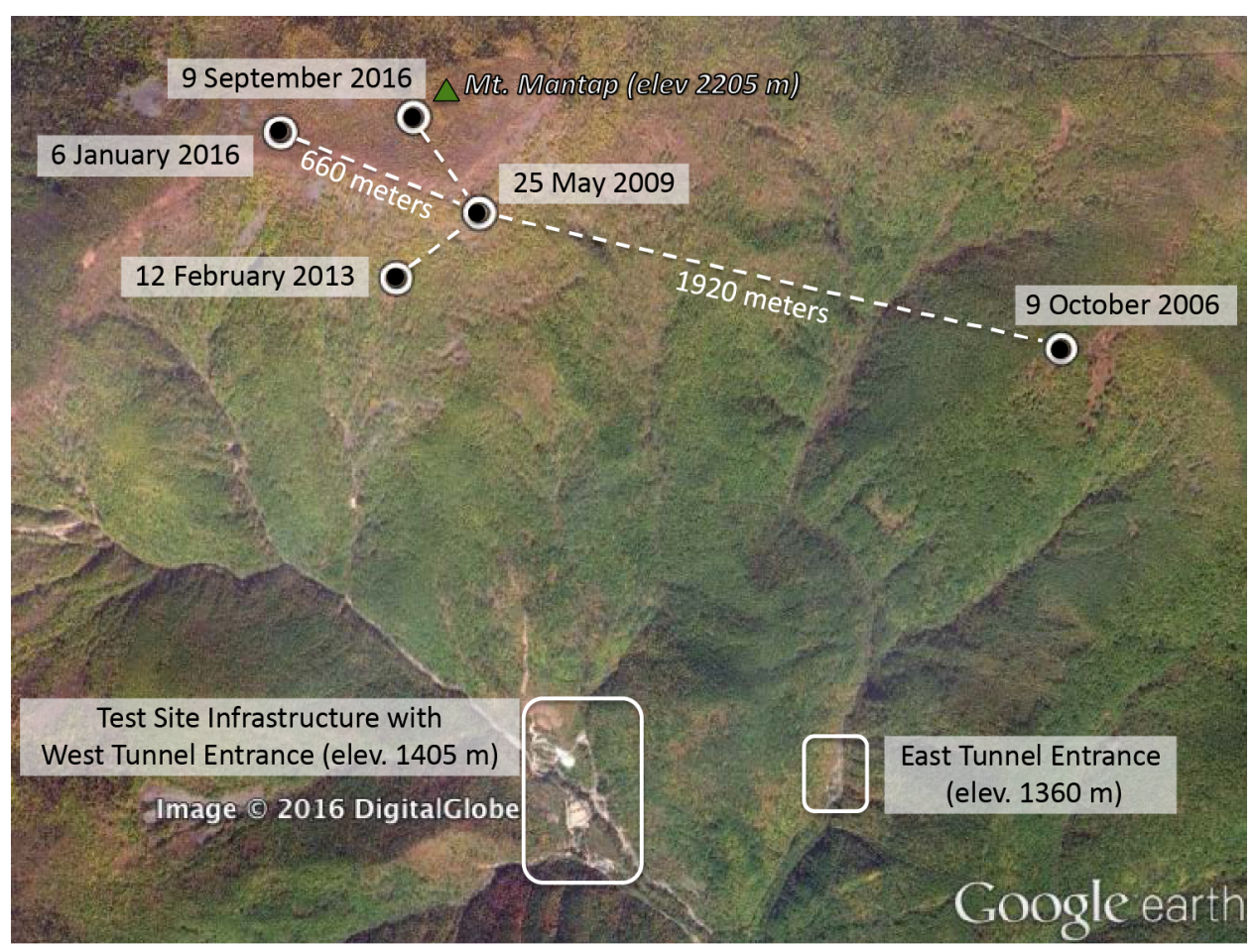

Figure 10. Commercial satellite image (from 18 September 2014) of the Punggye-ri test-site region near Mt. Mantap (as viewed on Google Earth), with a possible anchoring of the relative location estimates taking into consideration the ground infrastructure and assuming that maximizing the available overburdens was a test engineering priority. The coordinates of the proposed 2006 test hypothesis are $41.2904^{\circ} \mathrm{N}, 129.1039^{\circ} \mathrm{E}$ (elevation 1920 meters) and the corresponding coordinates for the January 2016 event are $41.2964^{\circ} \mathrm{N}, 129.0793^{\circ} \mathrm{E}$ (elevation 2189 meters). Any small lateral translation of this template (within a few hundred meters) is consistent with the seismic data presented in this study. A translation to the South would reduce the lengths of the necessary tunnels but also reduce the overburden. 